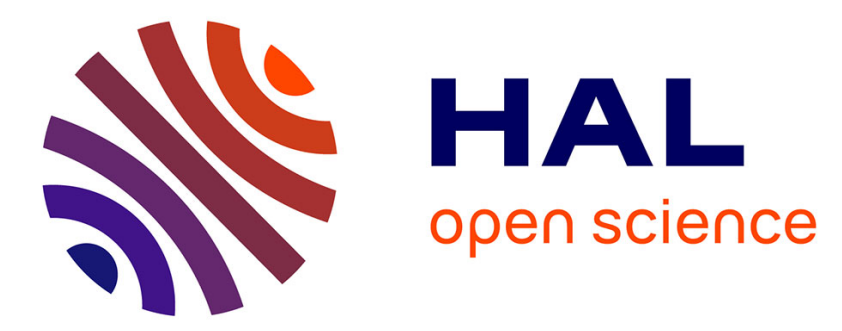

\title{
Evolution des paysages et anthropisation depuis le Néolithique dans la péninsule de La Hague (Normandie, France)
}

L. Lespez, M. Clet-Pellerin, Robert Davidson, Cyril Marcigny, B. Hardel, F. Levalet

\section{To cite this version:}

L. Lespez, M. Clet-Pellerin, Robert Davidson, Cyril Marcigny, B. Hardel, et al.. Evolution des paysages et anthropisation depuis le Néolithique dans la péninsule de La Hague (Normandie, France). Archeosciences, revue d'Archéométrie, 2004, 28 (28), pp.71-88. 10.3406/arsci.2004.1063 . hal00283551

\section{HAL Id: hal-00283551 \\ https://hal.science/hal-00283551}

Submitted on 10 Feb 2021

HAL is a multi-disciplinary open access archive for the deposit and dissemination of scientific research documents, whether they are published or not. The documents may come from teaching and research institutions in France or abroad, or from public or private research centers.
L'archive ouverte pluridisciplinaire $\mathbf{H A L}$, est destinée au dépôt et à la diffusion de documents scientifiques de niveau recherche, publiés ou non, émanant des établissements d'enseignement et de recherche français ou étrangers, des laboratoires publics ou privés.

\section{(ㅇ)(1) $\$$}

Distributed under a Creative Commons Attribution - NonCommercial - NoDerivatives| 4.0 


\section{Évolution des paysages et anthropisation depuis le Néolithique} dans la péninsule de La Hague (Normandie, France)

Laurent Lespez, Martine Clet-Pellerin, Robert Davidson, Cyril Marcigny, Blandine Hardel, François Levalet

\section{Citer ce document / Cite this document :}

Lespez Laurent, Clet-Pellerin Martine, Davidson Robert, Marcigny Cyril, Hardel Blandine, Levalet François. Évolution des paysages et anthropisation depuis le Néolithique dans la péninsule de La Hague (Normandie, France). In: Revue d'Archéométrie, $\mathrm{n}^{\circ} 28,2004$. pp. 71-88;

doi : https://doi.org/10.3406/arsci.2004.1063

https://www.persee.fr/doc/arsci_0399-1237_2004_num_28_1_1063

Fichier pdf généré le 24/10/2018 


\title{
Résumé
}

Les recherches paléoenvironnementales entreprises dans la péninsule de La Hague contribuent à la connaissance de des paysages océaniques du nord-ouest français dans une région restée longtemps à l'écart des études paléoenvironnementales sur l'Holocène. L'inventaire systématique des archives naturelles disponibles a permis d'identifier plusieurs espaces d'investigations comme une petite plaine littorale et des fonds de vallées. Les remplissages holocènes, épais de 2 à $4 \mathrm{~m}$, montrent des séquences tourbeuses et des apports détritiques. Les sondages, réalisés à la tarière et à la sonde russe, sont positionnés entre eux au sein d'un SIG par l'utilisation du GPS différentiel. Les analyses sédimentologiques et palynologiques permettent de caractériser l'évolution des dynamiques fluviatiles et végétales alors que le contrôle chronologique est assuré par des datations radiocarbone.

Le remplissage tourbeux débute il y a 4500 ans BP dans la petite plaine littorale mais plus tard dans les fonds de vallée (2000 BP). Les premiers signaux indiquant l'anthropisation des paysages apparaissent à la charnière Néolithique final - âge du Bronze. Cependant, il faut attendre la deuxième partie de l'âge du Fer puis la période gallo-romaine pour que s'imposent les pratiques agro-pastorales qui engendrent l'ouverture conséquente du milieu forestier. Localement le développement de landes basses régressives est daté de l'époque médiévale en relation avec la dégradation des profils pédologiques. Cette évolution atteste l'ancienneté des paysages océaniques de La Hague et le rôle fondamental des sociétés dans leur mise en place.

\begin{abstract}
The palaeoenvironmental researches conducted in the Hague peninsula contribute to the knowledge of the landscapes changes of Northwestern France. A small coastal plain and valleys bottoms were particularly investigated by field work and drillings. The alluvial and landscape changes are determined on the basis of sedimentological, micromorphological and palynological analyses. The dating control is given by radiocarbon dating and archaeological remains.

In the coastal plain, the peaty aggradation begun around 4500 yrs BP whiles in the valleys it begun later around $2000 \mathrm{yrs}$ BP. The first indices of clearance appear at the cusp of Neolithic and Bronze Age. Nevertheless, the final forest clearing become latter, during the Late Iron Age and the Galloroman period, in relation with the land use and cattle breeding increase. Locally, the relationship between the impoverishment of the soils and the development of current healthland is testified.
\end{abstract}




\title{
Evolution des paysages et anthropisation depuis le Néolithique dans la péninsule de La Hague (Normandie, France)
}

\author{
Laurent LESPEZ*, Martine CLET-PELLERIN**, Robert DAVIDSON*, Cyril MARCIGNY****, \\ Avec la collaboration de Blandine HARDEL* et François LEVALET*
}

\begin{abstract}
Résumé : Les recherches paléoenvironnementales entreprises dans la péninsule de La Hague contribuent à la connaissance de l'évolution des paysages océaniques du nord-ouest français dans unc région restée longtemps à l'écart des études paléoenvironnementales sur l'Holocènc. L'inventaire systématique des archives naturelles disponibles a permis d'identifier plusieurs espaces d'investigations comme une petite plainc littorale et des fonds de vallées. Les remplissages holocèncs, épais de 2 à $4 \mathrm{~m}$, montrent des séquences tourbeuses et des apports détritiques. Les sondages, réalisćs à la tarière et à la sonde russe, sont positionnés entre eux au scin d'un SIG par l'utilisation du GPS différentiel. Les analyses sédimentologiques et palynologiques permettent de caractériser l'évolution des dynamiques fluviatiles et végétales alors que le contrôle chronologique est assuré par des datations radiocarbonc.

Le remplissage tourbcux débute il y a 4500 ans BP dans la petite plaine littorale mais plus tard dans les fonds de valléc (2000 BP). Les premicrs signaux indiquant l'anthropisation des paysages apparaissent à la charnic̀re Néolithique final - âge du Bronze. Cependant, il faut attendre la deuxième partic de l'âge du Fer puis la période gallo-romaine pour que s'imposent les pratiques agro-pastorales qui cngendrent l'ouverture conséquente du milieu foresticr. Localement le développement de landes basses régressives est daté de l'époque médiévale en relation avec la dégradation des profils pédologiques. Cette évolution atteste l'ancienneté des paysages océaniques de La Hague et le rôle fondamental des sociétés dans leur mise en place.
\end{abstract}

Abstract : The palaeoenvironmental researches conducted in the Hague peninsula contribute to the knowledge of the landscapes changes of Northwestern France. A small coastal plain and valleys bottoms were particularly investigated by field work and drillings. The alluvial and landscape changes are determined on the basis of sedimentological, micromorphological and palynological analyses. The dating control is given by radiocarbon dating and archaeological remains.

In the coastal plain, the peaty aggradation begun around 4500 yrs BP whiles in the valleys it begun later around 2000 yrs BP. The first indices of clearance appear at the cusp of Neolithic and Bronze Age. Nevertheless, the final forest clearing become latter, during the Late Iron Age and the Gallo-roman period, in relation with the land use and cattle breeding increase. Locally, the relationship between the impoverishment of the soils and the development of current healthland is testified.

Mots-clés : Plaines littorales, sédimentation alluviale, landes, Holocène, Massif Armoricain.

Key-words : Coastal plain, alluvial records, moorland, Holocene, Western France.

\section{Introduction}

Depuis le travail fondateur de $\mathrm{H}$. Elhaï (1963), les recherches sur l'Holocène normand prennent un nouvel essor. Elles sont relancées dans le cadre de programmes pluridisciplinaires à la croisée des sciences des paléoenvironnements, de l'archéologie et de l'histoire. La péninsule de La Hague, pointe occidentale du Cotentin (fig. 1) est une région particulièrement propice à ces nouvelles investigations. Marquée par un climat océanique et des paysages armoricains caractérisés par l'importance des landes littorales, cet espace original possède des affinités avec l'ensemble des «Finistères» de la façade occidentale de l'Europe. Il représente, comme les îles anglo-normandes (Campbell, 2000 ; Campbell et al., 2001), un jalon entre les parties occidentales et méridionales du massif armoricain, au sud (Elhaï, 1963 ; Visset, 1979 ; Gebhardt, 1990 ; Marguerie, 1992 ; Billard et al., 1995 ; Barbier, 1999) et le sud-ouest de l'Angleterre, au nord de la Manche (Caseldine, 1999 ; Gearey et al., 2000a, b ; Waller et Long, 2003) (fig. 1).

Le but de cet article est de présenter l'évolution des paysages et les interactions Nature/Sociétés depuis le Néolithique. L'importance du remplissage

*GEOPHEN-LETG-UMR 6554 CNRS, Université de Caen-Basse Normandie, Esplanade de la Paix, BP 5186, 14032 CAEN cedex. laurent.lespez@geo.unicacn.fr et davidson@gco.unicaen.fr

**M2C-UMR 6143 CNRS, Université de Caen-Basse Normandie, 24 rue des Tilleuls, 14000 CAEN. martinc.clet@geos.unicacn.fr ***GEOPHEN-LETG-UMR 6554 CNRS, Université de Caen-Basse Normandie, Esplanade de la Paix, BP 5186, 14032 CAEN cedex. ****INRAP, Boulevard de L'Europe, 14540 BOURGUÉBUS, cyril.marcigny@wanadoo.fr 

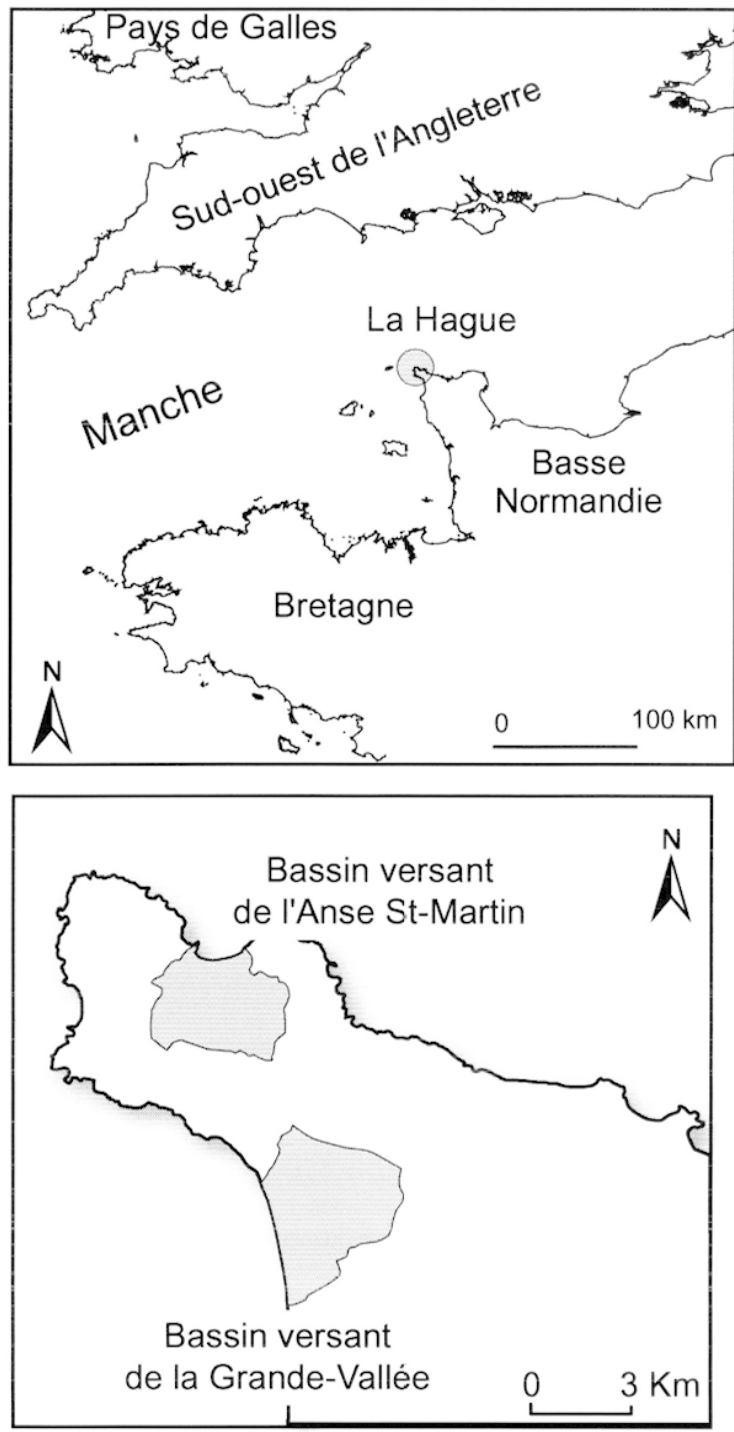

Figure 1 : Localisation des cspaces ctudićs. Figure 1 : Study areas.

sédimentaire des fonds de vallées et des petites plaines littorales offre un potentiel intéressant pour les études paléoenvironnementales sur l'Holocène (Lespez et al., 2003) alors qu'une implantation humaine est attestée depuis le Mésolithique (Letterlé et Verron, 1986 ; Chancerel et al., 1996 ; Ghesquière et al., 2000). Jusqu'à présent les paléoenvironnements du Pléistocène supérieur étaient mieux connus du fait des études sur le peuplement paléolithique (Cliquet, 1994). Mais les fouilles en cours du site archéologique de «la Jupinerie» à Omonville-laPetite occupé temporairement du Néolithique moyen au moins, au Bronze final (Marcigny et al., 2002, 2003) et les recherches sur le moulin de Vauville (Lespez et al., 2003) ouvrent de nouveaux horizons sur la connaissance des environnements holocènes.

\section{Le terrain d'étude}

\subsection{Les unités paysagères}

La péninsule de La Hague comporte quatre unités paysagères principales :

- Des enclos bocagers, limités par des murets de pierres sèches ou des haies végétales (hêtres, aubépines, ronces), qui possèdent une vocation principalement herbagère même si la culture du maïs s'est développée au cours des 20 dernières années. Ils occupent principalement les sols bruns acides des plateaux schisto-gréseux (100 à $180 \mathrm{~m}$ d'altitude) et les sols bruns plus ou moins lessivés et bien ressuyés des terrasses marines pléistocènes, des marges des plaines littorales et des fonds de vallées.

- Des landes qui caractérisent de nombreux versants en particulier en position littorale et constituent encore quelques grands ensembles comme dans le bassin versant de la Grande-Vallée à Vauville.

- Des bois résiduels (hêtres, chênes, châtaigniers, saules, aulnes, noisetiers, frênes) qui se développent principalement sur les versants accidentés qui dominent de 60 à $100 \mathrm{~m}$ les fonds des vallées étroites qui découpent le plateau.

- Des zones humides et des marais arborés (saules, aulnes) ou couverts de roseaux qui caractérisent certains fonds de vallées et les petites plaines littorales.

\subsection{Les paysages de landes}

Les paysages de landes sont la marque la plus évidente de l'aspect océanique de La Hague. Ils sont d'une grande diversité (Provost et Lecointe, 1985) et se développent sur des sols minces le plus souvent de type ranker ou podzol humo-ferrugineux. Les landes littorales sont le plus souvent des landes sèches hautes à ajoncs d'Europe (Ulex europaeus) et fougères aigles (Pteridium aquilinum=Pteris a.) avec quelques plaques de landes à bruyères cendrées (Erica cinerea). Les landes basses occupaient également une place importante (Landes de Jobourg, de Beaumont...) mais sont aujourd'hui principalement limitées au bassin versant de la Grande-Vallée de Vauville (Hauguel et Lecointe, 1996). Elles sont dominées par les ajoncs nains (Ulex gallii), les bruyères cendrées (Erica cinerea) et les callunes (Calluna vulgaris). Dans les fonds de vallées, caractérisés par l'hydromorphie, prennent place des landes tourbeuses à bruyères quatre angles (Erica tetralix), comportant des ajoncs, des callunes, des joncs et des touradons de molinie (Molinia caerulea) mais également des saules (Salix).

\subsection{Deux bassins versants complémentaires}

Dans ce contexte morpho-climatique, les lieux d'enregistrement des dynamiques sédimentaires et végétales holocènes sont constitués par les fonds de vallées, les plaines littorales et les tourbières de plateaux. Ces dernières forment des espaces privilégiés et ont été abondamment exploitées dans le Massif Armoricain et le sudouest de l'Angleterre (Visset, 1979; Marguerie, 1992 ; Barbier, 1999; Edwards, 1999). Malheureusement les tourbières ombrogènes et soligènes de tête de versant n'existent plus à La Hague depuis la construction du centre de retraitement des déchets nucléaires; il a donc fallu s'appuyer sur l'étude sur des tourbières topogènes et fluviogènes. Ces tourbières minérotrophes possèdent deux avantages : une sédimentation organique et détritique favorable à l'analyse palynologique et à l'étude des transferts des sédiments dans l'ensemble des bassins ver- 
sants et souvent une plus grande proximité des espaces peuplés et mis en valeur comme l'atteste de nombreuses recherches (Leroyer, 1997 ; Brown, 1999, par exemple).

Deux bassins versants complémentaires ont été étudiés (fig. 1). L'Anse Saint-Martin constitue le principal rentrant sur la côte nord de La Hague entre la pointe de Jardeheu à l'est et le cap de La Hague à l'ouest. Au pied des anciennes landes de Beaumont et de Jobourg aujourd'hui occupées par le centre de retraitement des déchets nucléaires (COGEMA), elle est bordée par un cordon de galets qui protège une plaine littorale occupée par des prairies humides et un petit marais comportant une étendue d'eau libre en hiver, une ceinture de roseaux, puis de saules et d'aulnes. Lors des grandes marées, en période de démaigrissement du cordon littoral, une tourbière fossile peut être observée sur l'estran actuel. Contrairement à d'autres formations tourbeuses situées dans des contextes semblables sur le littoral de Basse-Normandie (Elhaï, 1963 ; Clet-Pellerin, 1985 ; Clet-Pellerin et al., 1987), elle n'avait pas fait l'objet d'analyses et sa genèse comme son âge demeuraient inconnus. Située à moins d'un kilomètre du site de «La Jupinerie», son étude offre l'opportunité de comprendre l'environnement dans lequel se sont installées les populations et de préciser la formation des paysages au cours des cinq derniers millénaires. Son analyse a été complétée par celle du remplissage du fond de vallée de la Sainte Hélène, principal cours d'eau du bassin versant de l'Anse Saint Martin.

La Grande-Vallée de Vauville constitue le coeur du plus vaste ensemble de landes basses de La Hague (700 ha). Une zone de sédimentation organique el détritique en arrière d'un ancien barrage associé au bief et au moulin de Vauville, a permis de déterminer l'évolution des paysages de fond de vallée associée aux transformations hydrauliques et agraires des deux derniers millénaires.

\section{Méthodologie}

L'inventaire systématique des archives naturelles disponibles par plus de soixante sondages a permis de préciser l'ampleur et la géographie des remplissages holocènes. Epais de 1,5 à 3,5 m, au moins, ils montrent des séquences organiques et des apports détritiques. Les sondages, réalisés à la tarière à main et à la sonde russe, sont positionnés entre eux par l'utilisation d'un GPS différentiel qui permet de construire une base de données géoréférencées sous la forme d'un SIG et de réaliser des restitutions précises du remplissage sédimentaire. Sept sondages ont fait l'objet d'un prélèvement systématique pour des analyses sédimentologiques, micromorphologiques et palynologiques afin de caractériser les dynamiques fluviatiles et végétales. Les analyses granulométriques de 3 sondages (SM $60,13,11$; fig. 2) ont été réalisées au granulomètre laser (Beckman-Coulter LS 200), après destruction de la matière organique, à partir d'une maille de prélèvement de 5 à $10 \mathrm{~cm}$. Dans la Grande-Vallée de Vauville, sur un sondage réalisé à la pelle mécanique (VAU 76), cinq lames minces «grand format» $(14 \times 6 \mathrm{~cm})$ ont fait l'objet d'analyses micromorphologiques.

L'étude palynologique porte sur 3 sondages, de 1,2 à 3,5 mètres de profondeur, réalisés au carottier russe (SM 60, SM 2/03et VAU 76). Les échantillons ont été prélevés selon une maille de 5 à $10 \mathrm{~cm}$. La description des résultats de l'analyse pollinique s'appuie sur un diagramme pollinique classique

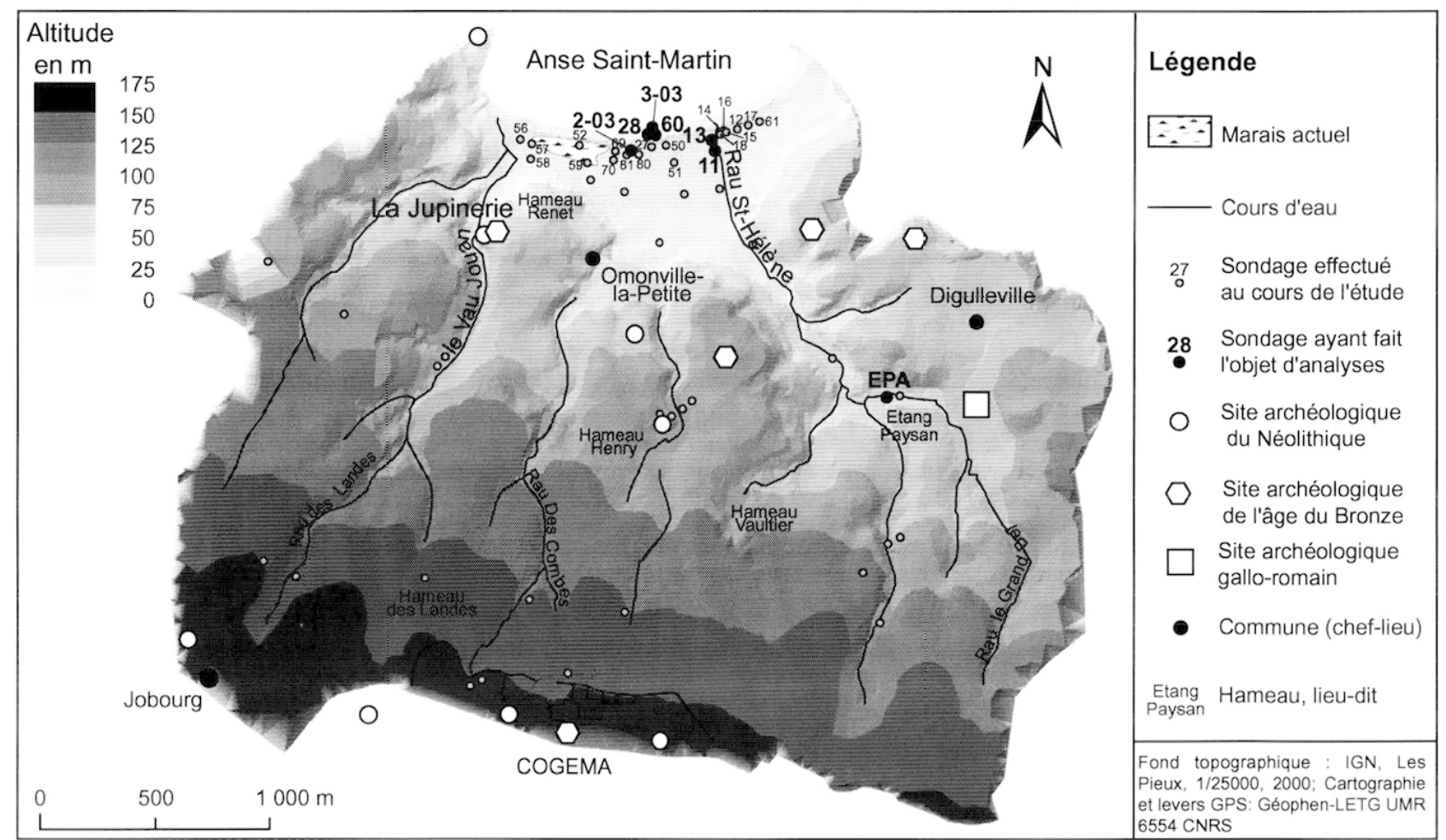

Figure 2 : Environnement oro-hydrographique et localisation des sondages réalisés et des sites archéologiques connus dans le bassin versant de l'Anse Saint-Martin.

Figure 2: Bore holes, core drillings and archaeological sites location in the Anse Saint Martin river basin. 
et sur la comparaison de ces différents diagrammes. Un diagramme de comparaison est établi à partir des courbes de pourcentages de taxons ou de groupements de taxons représentatifs, calculés sur la somme totale des grains de pollen et spores comptés amputée des pourcentages des spores monolètes (colonisatrices d'espaces après assèchement de zone humide), des plantes de milieu aquatique (Typha, Sparganium, Myriophyllum, Nuphar, Alisma plantago, Polygonum amphibium...), et des plantes caractéristiques des schorres (Chenopodiaceae, Plantago maritima, Armeria...). Huit groupes de taxons ou de groupements de taxons sont représentés : les taxons forestiers avec les courbes de Quercus, Corylus et Alnus ; le groupement des taxons herbacés de zones humides ou marécageuses avec les Cyperaceae, Filipendula, Epilobium, Thalictrum, Phragmites, Osmunda ; le groupement des taxons de landes avec les fougères (Pteris), les Ericaceae (Calluna, Erica) et les Fabaceae ; le groupement des taxons de plantes rudérales avec les friches : Asteraceae (Tubuliflorae et Liguliflorae), Brassicaceae, Caryophyllaceae, Rumex et les zones piétinées : Lamiaceae, Urticaceae, Artemisia ; le groupement des taxons de prairies et de pâtures avec les Poaceae, Apiaceae, Ranunculaceae, Plantago lanceolata; et le groupement des taxons de plantes cultivées (Cerealia, Fagopyrum) et de plantes messicoles accompagnatrices (Papaveraceae, Centaurea cyanus...).

Le contrôle chronologique est assuré par dix datations radiocarbone conventionnelles établies au Centre d'Etudes Nordiques de Québec (Canada) et deux datations AMS à l'Institut de Physique d'Erlangen (Allemagne) calibrées à 2 écart-type (tab. 1) et par des éléments archéologiques retrouvés dans les sondages ou des informations historiques sur les espaces de sédimentation.

\section{Les analyses paléoenvironnementales}

\subsection{L'Anse Saint-Martin}

La plaine littorale de l'Anse Saint-Martin protégée par un cordon de galets comporte une tourbière caractérisée par une roselière et des bosquets de saules alors que sur ses marges se développent des prairies humides. Sur l'estran apparaît ponctuellement une sédimentation tourbeuse qui montre plusieurs faciès : tourbe à macrorestes végétaux (roseaux principalement) et tourbe à bois fossiles. L'ensemble, d'une épaisseur comprise entre 2 et $4 \mathrm{~m}$, a pu être étudié grâce à de nombreux sondages. Les

\begin{tabular}{|c|c|c|c|c|c|c|}
\hline Site & $\begin{array}{c}\text { Code } \\
\text { laboratoire }\end{array}$ & Sondage & $\begin{array}{l}\text { Profondeur } \\
\text { (m) }\end{array}$ & Matériel & $\begin{array}{c}\text { Dates BP } \\
\qquad(1 \sigma)\end{array}$ & $\begin{array}{l}\text { Dates calibrées } \\
\qquad(2 \sigma)\end{array}$ \\
\hline Anse Saint-Martin & UL-2354 & SM 60 & $2,60-2,65$ & Limons organiques & $4510 \pm 100$ & 3517-2905 av. J.-C. \\
\hline Anse Saint-Martin & UL-2364 & SM 60 & $1,60-1,65$ & Limons organiques & $3560 \pm 100$ & 2196-1638 av. J.-C. \\
\hline Anse Saint-Martin & UL-2313 & SM 60 & $0,20-0,30$ & Tourbe & $1780 \pm 70$ & $77-419$ ap. J.-C. \\
\hline Anse Saint-Martin & Erl-6466 & SM 2-03 & $2,40-2,44$ & Limons organiques & $2571 \pm 59$ & 832-413 av. J.-C. \\
\hline Anse Saint-Martin & UL-2762 & SM 2-03 & $1,69-1,79$ & Tourbe & $1470 \pm 80$ & 414690 ap. J.-C. \\
\hline Anse Saint-Martin & UL-2763 & SM 2-03 & $0,87-0,94$ & Tourbe & $240 \pm 80$ & $1480-1950$ ap. J.-C. \\
\hline Anse Saint-Martin & UL-2404 & SM 28 & $2,27-2,36$ & Limons organiques & $4310 \pm 80$ & $3259-2698$ av. J.-C. \\
\hline Anse Saint-Martin & UL-2400 & SM 14 & $0,45-0,50$ & Tourbe & $1720 \pm 90$ & 84-539 ap. J.-C. \\
\hline Anse Saint-Martin & Erl-6465 & SM 3-03 & 2,60 & Charbon & $4154 \pm 58$ & 2883-2579 av. J.-C. \\
\hline Sainte-Hélène & ErL-2556 & EPA & $1,9-1,93$ & Tourbe & $2150 \pm 90$ & 397 av. -50 ap. J.-C \\
\hline Vauville & UL-2554 & Vau & $1,05-1,15$ & Tourbe & $1550 \pm 90$ & 261-660 ap. J.-C. \\
\hline Vauville & UL-2565 & Vau & $0,60-0,70$ & Tourbe & $910 \pm 60$ & 1003-1260 ap. J.-C. \\
\hline
\end{tabular}

Programme de calibration CALIB rev. 4.3. (Stuiver et al., 1998)

Tablcau 1: Datations radiocrabonc.

Table 1: Radiocarbone dating. 
sondage SM 60 et SM 2/03 ont fait plus particulièrement l'objet d'investigations sédimentologiques et palynologiques (fig. 2).

\subsubsection{Stratigraphie et analyses sédimentologiques}

\section{Chronologie du remplissage sédimentaire}

Dans l'Anse Saint-Martin comme dans le reste du Nord-Cotentin (Coutard, 2003 ; Coutard et Clet-Pellerin, à paraître), la sédimentation holocène débute par une sédimentation argileuse bleu gris sans doute d'origine marine (marais maritime). Puis, au cours de la deuxième partie de l'Holocène, la progradation du cordon de galets vers le large entraîne le développement d'une sédimentation continentale caractéristique d'une plaine d'inondation en partie marécageuse (fig. 3). Dans l'Anse Saint-Martin, les trois datations radiocarbone obtenues à la base de la sédimentation tourbeuse sur l'estran actuel montrent que la sédimentation tourbeuse a débuté entre 4500 et 4100 BP $(4510+/-100$ BP sur le sondage SM $60 ; 4310$ $+/-80$ BP sur le sondage SM 28 et $4154+/-58$ BP sur le sondage SM 3-03). Ensuite le recul du trait de côte entraine la fossilisation de la tourbière littorale ancienne sous le cordon de galets et l'estran actuel et, en arrière, le développement d'un bas-marais tourbeux alimenté par les cours d'eau qui descendent des landes de Beaumont et de Jobourg.
Nature et répartition spatiale du remplissage sédimentaire

Les datations radiocarbone obtenues sur les sondages SM 60 et SM 2/03 permettent de déterminer le rythme global de la sédimentation organique dans le bas-marais fossile d'estran comme dans celui encore actif actuellement. Il a été très stable, de $0,7-0,8 \mathrm{~mm} / \mathrm{an}$, et permet d'estimer l'âge des principales ruptures sédimentaires ou palynozones. Pour la tourbière d'estran, si on prolonge le rythme de la séquence tourbeuse finale, la fin de la sédimentation étudiée peut-être estimée à 1420 BP environ. Dans ces tourbières, l'influence de la mer fut toujours quasiment nulle comme le montrent les pourcentages très faibles de Chenopodiaceae $(<1 \%$, en général) obtenus par les analyses polliniques pour les cinq derniers millénaires.

La sédimentation organique des milieux de bas-marais qui s'amorce à partir de 4500-4100 BP s'accompagne d'apports détritiques réguliers issus de l'ensemble du bassin versant comme l'indiquent les analyses granulométriques. La teneur en sables et limons des sédiments du sondage SM 60 (fig. 4) montre des apports fluviatiles réguliers de type limons de débordement (Grain moyen > $25 \mu \mathrm{m}$ ), alors que l'existence de passées de sables fluviatiles parfois relativement bien triés (So $<2,5$ ) caractéristiques de dépôts de chenaux, attestent l'existence de défluviations récurrentes de la Sainte-Hélène. Cela témoigne

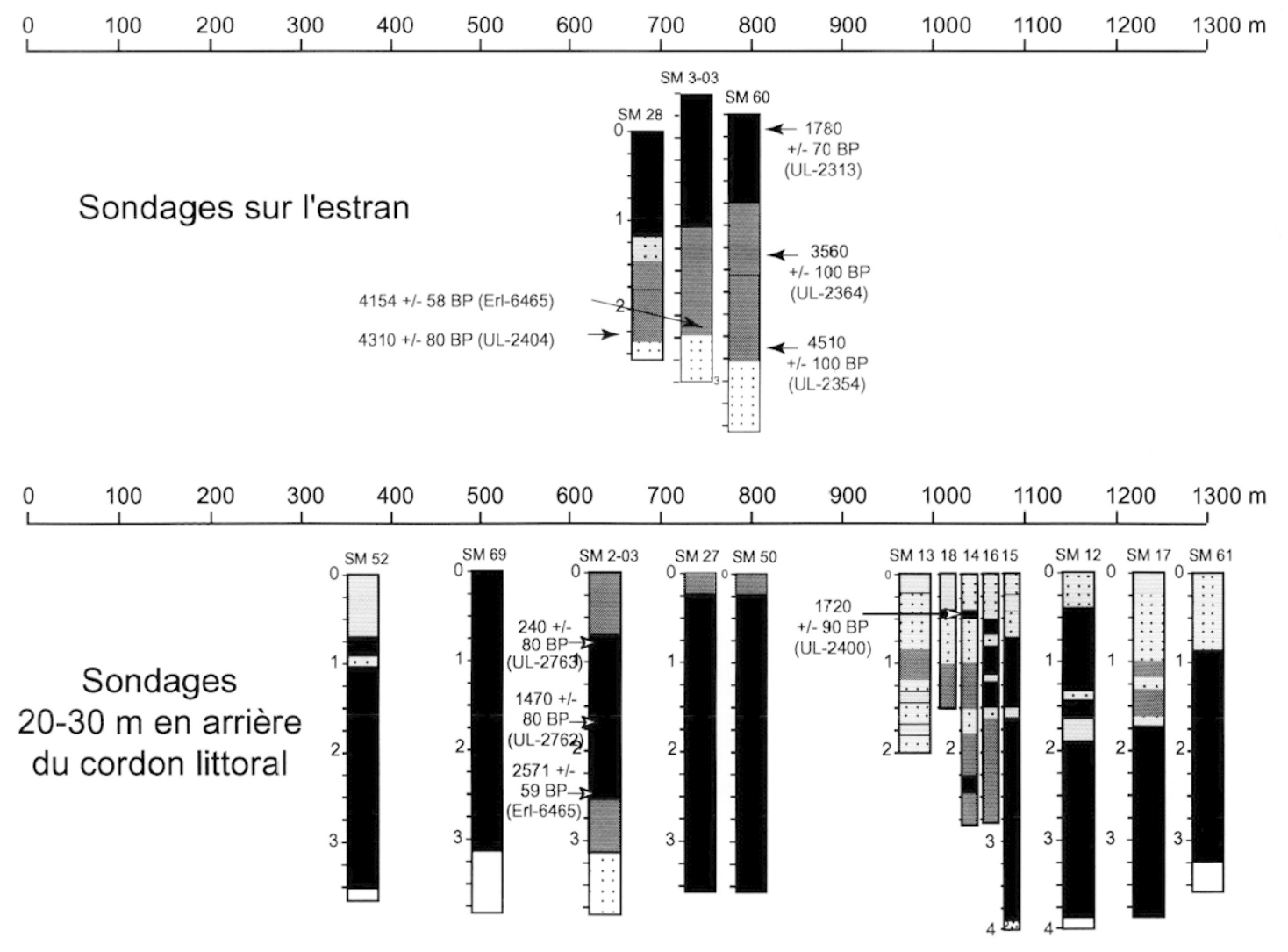

Echelle verticale en mètres

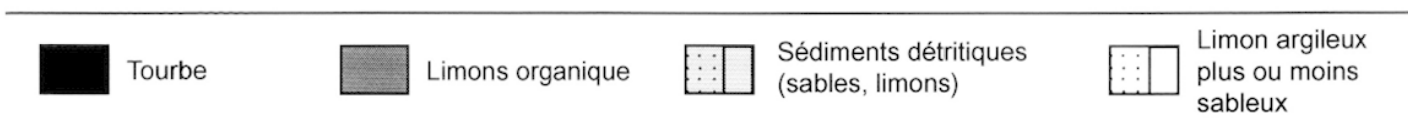

Figure 3 : Formations superficielles de la plaine littoralc de l'Anse Saint-Martin.

Figure 3 : Late Holocene alluvial filling in the Anse Saint Martin coastal plain. 


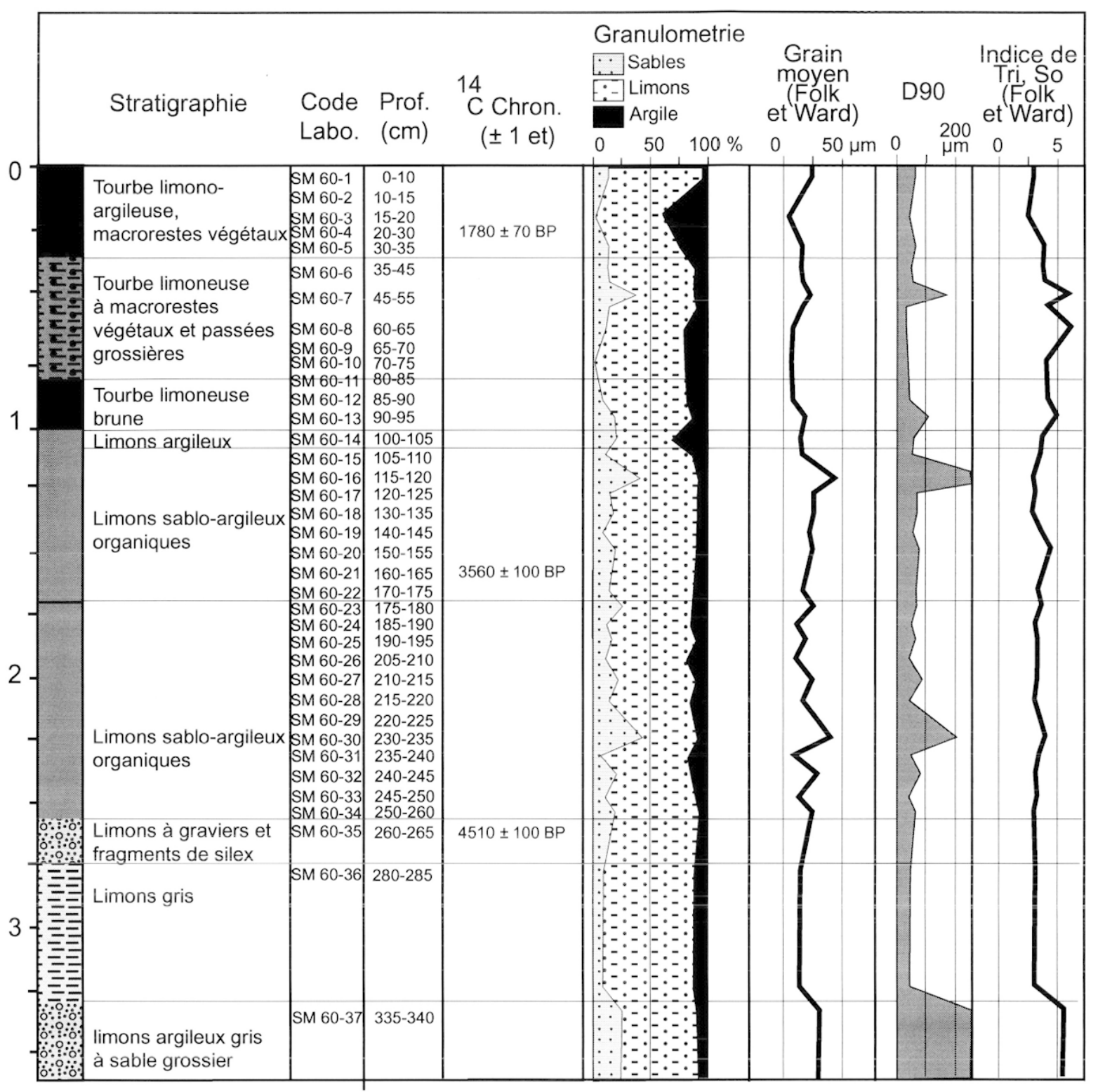

Analyses granulométriques : Geophen-UMR LETG 6554 CNRS; Datations radiocarbone : Centre d'études nordiques, Quebec

Figure 4 : Les analyses granulométriques du sondage SM 60. Figure 4 : Grain Size analysis - SM 60.

d'une accentuation des flux solides dans le bassin versant de l'Anse Saint-Martin dès l'Holocène moyen. Vers l'Est de la plaine littorale, au-dessus de deux mètres de profondeur, la sédimentation organique devient de plus en plus détritique et indique des apports fluviatiles responsables de la construction d'un petit cône alluvial entraînant l'atterrissement progressif des milieux palustres. Cet atterrissement s'est généralisé au cours des deux derniers millénaires comme en témoignent les différents sondages effectués dans la partie orientale du marais et une date radiocarbone obtenue vers le sommet de l'un d'entre eux (SM $14: 1720+/-90$ BP, fig. 3).

3.1.2. Les analyses palynologiques de la séquence organique d'estran (Sondage SM 60)

Les analyses palynologiques reposent sur l'examen de 71 échantillons répartis sur un sondage effectué sur l'estran (SM 60) et un sondage dans le bas-marais actuel (SM 2-03). Il est possible d'identifier 4 grandes zones dans la séquence d'estran (SM 60) qui se divisent ellesmêmes en sous-zones (fig. 5).

\section{Palynozone SM 60-1 $(260-170 \mathrm{~cm})$}

Trois sous-zones sont mises en évidence. A la base (SM 60-1a), les grains de pollen d'Alnus, de Corylus et de Quercus forment l'essentiel de l'enregistrement pollinique. Le bas-marais est donc globalement occupé par une aulnaie alors que plus loin sur les marges ou sur les versants de la plaine littorale se développent les noisetiers et les chênes. Au-dessus (SM 60-1b), les pourcentages de grains de pollen d'Alnus et de Corylus diminuent progressivement alors que ceux des fougères augmentent légèrement. $\mathrm{Au}$ sommet du limon (SM 60-lc), les populations de Corylus sont en légère expansion mais on observe surtout une croissance des pourcentages de grains de pollen des Poaceae, de Cerealia, des plantes messicoles et de Pteris qui témoigne d'un premier développement des activités agricoles concomitant du développement des landes à fougère aigle. 


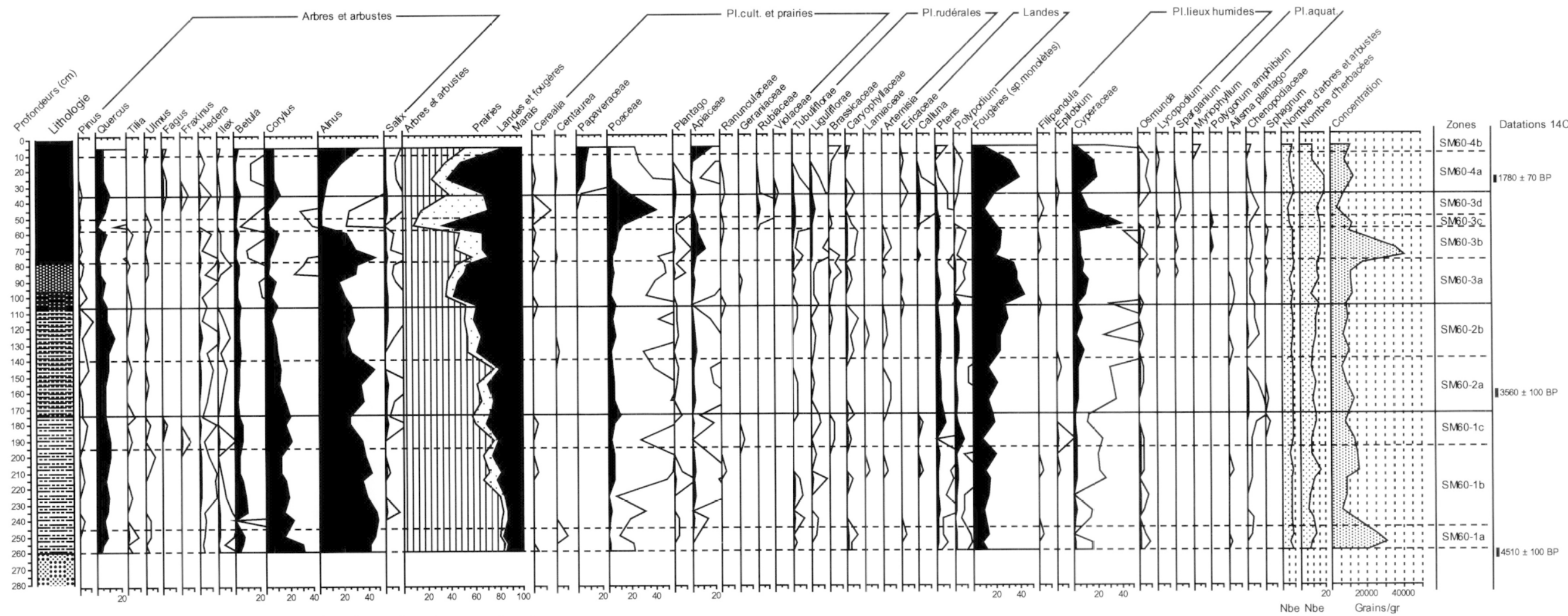

Figure 5 : Diagramme pollinique du sondage SM 60. Figure 5 : Pollen percentage diagram-SM 60. 


\section{Palynozone SM 60-2 $(170-105 \mathrm{~cm})$}

Tout au long de cette séquence le pourcentage des grains de pollen de Corylus diminue. Dans un premier temps le pourcentage des grains de pollen d'Alnus s'accroît (SM 60-2a) avant de diminuer pendant que Quercus augmente modérément (SM 60-2b). Mais dans son ensemble le couvert arboré et arbustif reste stable de même que les landes à fougères. Les traces d'activités agro-pastorales persistent mais restent faibles.

\section{Palynozone SM 60-3 (130-40 cm)}

Trois phases se succèdent. Dans un premier temps (SM 60-3a), après la brusque régression d'Alnus, le marais d'eau douce à Cyperaceae accompagné d'une ceinture de fougères commence à prendre de l'importance. Le paysage devient plus ouvert, la ceinture d'arbustes est plus éloignée du point d'échantillonnage, les pourcentages de grains de pollen d'herbacées (NAP) sont plus élevés que ceux des arbres (AP).

Ensuite (SM 60-3b), Alnus et Corylus s'étendent de nouveau et le paysage se referme légèrement dans le marais et sur ses marges. La concentration pollinique dans cette zone est élevée ce qui prouve qu'il n'y a pas de transport de pollen, la pluie pollinique se déposant sur place et provient de la végétation locale.

L'ouverture des paysages est ensuite plus nette (limite SM 60-3b, SM 60-3c). La chute brutale des pourcentages d'arbres et d'arbustes est d'abord liée à la montée de la nappe phréatique (chute d'Alnus) et à l'établissement d'un marais à Cyperaceae (SM 60-3c) puis à l'ouverture des paysages au-delà avec la baisse des pourcentages des grains de pollen de Quercus et de Corylus. Parallèlement, l'empreinte de l'homme est de plus en plus claire comme le montre le développement des pourcentages des grains de pollen de Cerealia, des graminées et des rudérales alors que la fougère aigle voit son importance relative diminuer au profit d'une lande basse à bruyère (SM 60-3d).

\section{Palynozone SM 60-4 $(40 \mathrm{~cm}-0 \mathrm{~cm})$}

On observe à nouveau l'extension d'une zone humide à Cyperaceae bordée de fougères alors que plus loin quelques zones boisées avec Corylus et Quercus subsistent (SM 60-4a). Au sommet de la palynozone (SM 60-4b), le paysage est toujours globalement ouvert et les activités agricoles attestées comme le montre le développement des plantes messicoles et rudérales mais les aulnes se développent de nouveau dans le centre humide de l'Anse alors que les essences pionnières (Betula et Corylus) recolonisent en partie les friches et la lande.

\subsubsection{Résultats de l'analyse palynologique de la} séquence organique du bas-marais actuel (Sondage SM 2/03)

Les analyses de ce sondage viennent compléter et étendre les analyses du sondage précédent. Il est possible d'identifier 4 grandes zones correspondant aux grands ensembles sédimentaires et qui se divisent elles-mêmes en sous-zones (fig. 6).
Palynozone SM 2/03-1 (244-220 cm)

La végétation est principalement composée de Cyperaceae et de fougères (spores monolètes) caractéristiques d'un environnement de zone humide. Les arbres et arbustes sont relativement rares autour du site. La concentration pollinique dépassant 20000 grains/cc indique une sédimentation sur place, sans transport de grains de pollen.

\section{Palynozone SM 2/03-2 $(220-110 \mathrm{~cm})$}

Les Phragmites accompagnent les Cyperaceae indiquant la permanence des milieux de bas-marais alors que le développement des plantes aquatiques implique une zone d'eau libre. Un fort développement des Poaceae et l'apparition de Cerealia indiquent le développement des activités agro-pastorales (SM 2/03-2a). Cependant, les pourcentages de grains de pollen d'arbres augmentent légèrement grâce au développement de Quercus, Corylus, Betula et Alnus. Le milieu se ferme lentement dans le marais (Alnus) et sur les pourtours de l'Anse (Quercus, Betula, Corylus) (SM 2/03-2a).

Ensuite, les pourcentages de grains de pollen d'arbres augmentent encore plus significativement (SM 2/03-2b). Une aulnaie s'installe en bordure des zones humides alors que les pourtours de l'Anse sont colonisés par d'autres arbres et arbustes (Corylus, Quercus et quelques Ulmus, Fagus). Les prairies sèches avec les Poaceae ou humides avec les Cyperaceae ainsi que les fougères diminuent d'importance.

Au sommet de l'épisode tourbeux, la végétation change à nouveau profondément (SM 2/03-2c). Les arbres disparaissent presque du paysage, sans doute à la suite de défrichements. Les prairies se développent à nouveau. $\mathrm{La}$ concentration est très élevée indiquant une sédimentation non perturbée.

\section{Palynozone SM 2/03-3 (110-46 cm)}

Depuis la base de cette zone, jusqu'au sommet du sondage, la végétation varie peu. Les plantes de prairies, les céréales, les plantes messicoles et rudérales occupent l'essentiel du paysage végétal. Sur les pourtours de l'Anse, quelques arbres et arbustes subsistent (Quercus, Corylus) (SM 2/03-3a). Dans un deuxième temps, les landes et les noisetiers se comportant en arbustes pionniers se développent (SM 2/03-3b).

\section{Palynozone SM 2/03-4 (46-5 cm)}

Les prairies sont toujours omniprésentes. La végétation subit peu de variations.

\subsection{La Grande-Vallée de Vauville}

Le fond de vallée a constitué un piège à sédiments organiques et détritiques dont l'épaisseur atteint au maximum 1,2 mètres (fig. 7). Les sondages montrent généralement la succession de trois séquences sédimentaires. A la base, une grave grossière sablo-graveleuse occupe l'ensemble $\mathrm{du}$ fond de vallée. Elle est surmontée par un sédiment tourbeux fréquemment recouvert par des atterrissements limono-sableux. 


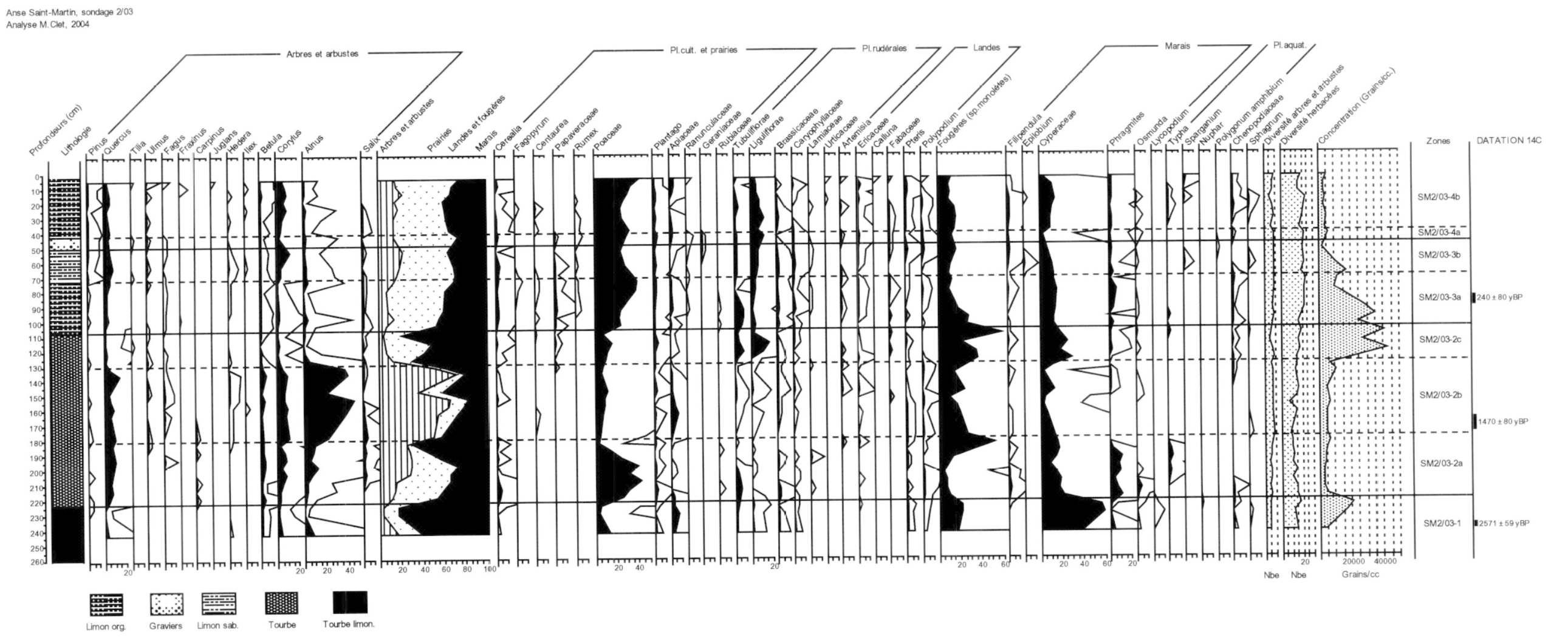

Figure 6 : Diagramme pollinique du sondage SM 2-03. Figure 6: Pollen percentage diagram-SM 2-03. 


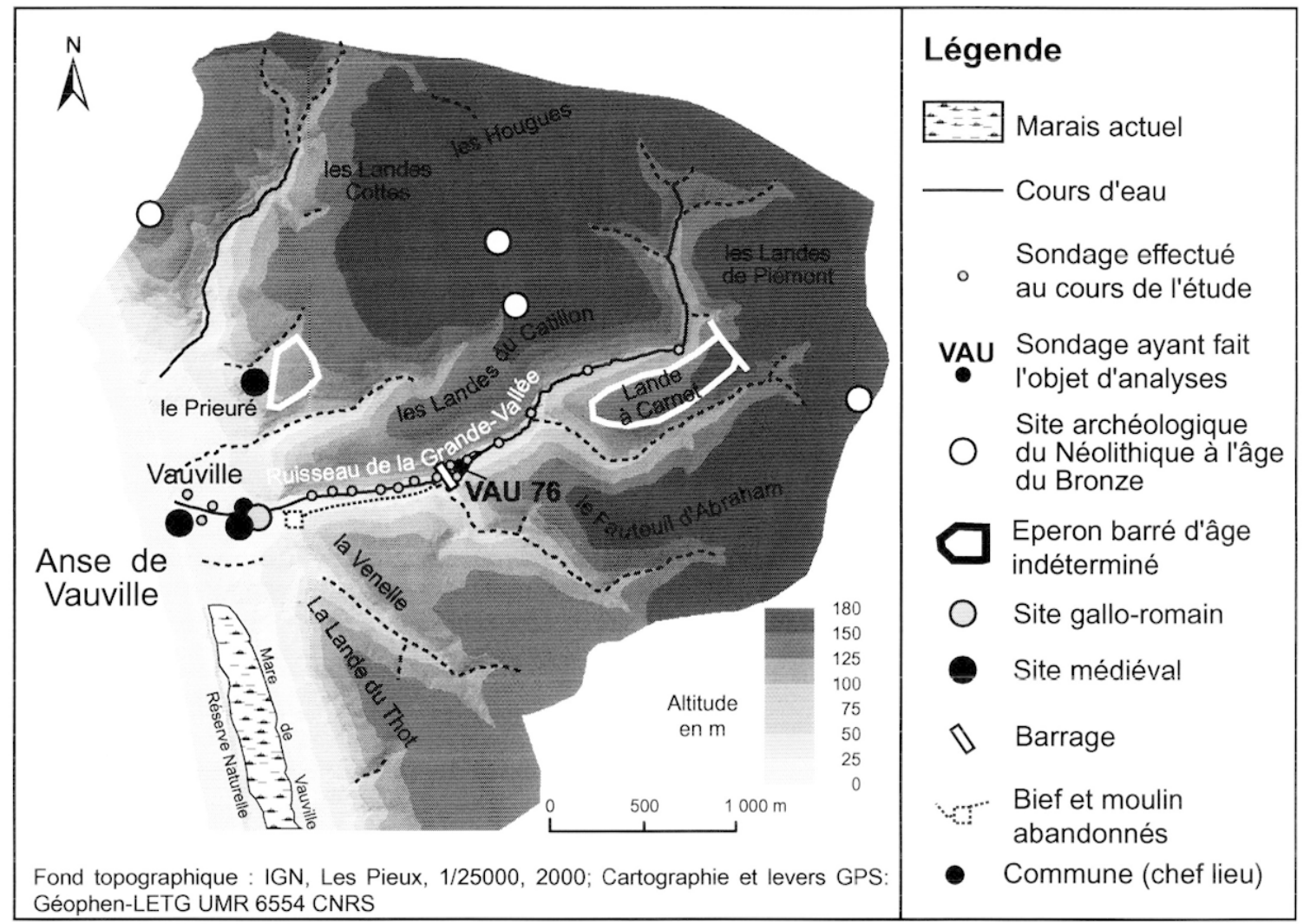

Figure 7 : Environnement oro-hydrographique et localisation des sondages et des sites archéologiques connus ct dans le bassin versant de la Grande Valléc de Vauville.

Figure 7 : Bore holes, core drillings and archaeological sites location in the Grande Vallée de Vauville river basin.

Les archives sédimentaires les plus dilatées sont observées en arrière du barrage de la Grande-Vallée aujourd'hui détruit. Un sondage (VAU 76) a fait l'objet d'analyses et révèle l'existence d'une stratigraphie plus complexe (fig. 8). Elle comporte une séquence de transition, entre les sédiments tourbeux et la séquence limono-sableuse, constituée de limons organiques. Elle montre également que la séquence détritique particulièrement épaisse localement $(60 \mathrm{~cm}$ par rapport au $30 \mathrm{~cm}$ observés ailleurs) est surmontée par un sol tourbeux, hydromorphe et riche en matière organique. Les formations tourbeuses de base se sont mises en place il y a 1500 ans environ comme l'indique une datation radiocarbone obtenue à la base du remplissage $(1550+/-90 \mathrm{BP})$. La fin de la sédimentation tourbeuse est localement datée de $910+/-60 \mathrm{BP}$ soit 1003-1260 ap. J.-C. Cette date correspond à la première mention d'un moulin à eau dans la Grande-Vallée en 1175 (Cartulaire de Vauville in Gérard, 1972). Les sédi-

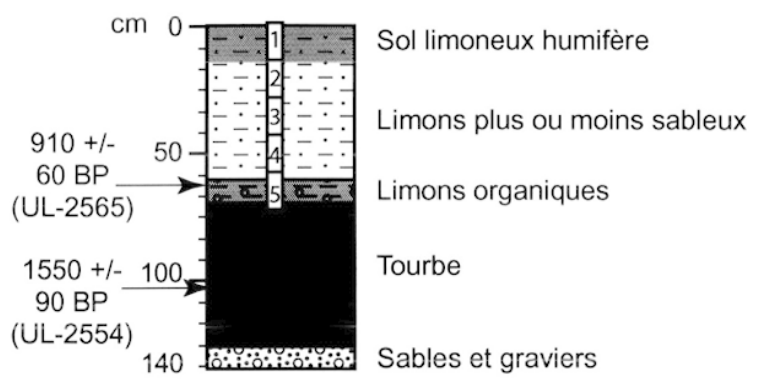

Figure 8: Chronostratigraphic et échantillons prélevés pour l'analyse micromorphologique sur le sondage VAU 76.

Figure 8: Chronostratigraphy and micromorphological samples - Vau 76. ments détritiques ont donc vraisemblablement été piégés, après les XII-XIII ${ }^{\mathrm{e}}$ siècles, derrière le barrage alimentant le bief du moulin.

\subsubsection{Les résultats de l'analyse palynologique du son-} dage VAU 76

Les analyses palynologiques portent principalement sur la séquence tourbeuse et sur les limons organiques de transition. Le rythme de sédimentation de la séquence tourbeuse de $0,7 \mathrm{~mm} / \mathrm{an}$ est identique à ceux observés à l'Anse Saint-Martin. Il est possible d'identifier 2 grandes zones corrcspondant aux grands ensembles sédimentaires et qui se divisent elles-mêmes en sous-zones (fig. 9).

\section{Palynozone VAU-1 $(100 \mathrm{~cm}-60 \mathrm{~cm})$}

De 100 à $87 \mathrm{~cm}$ (VAU-1a), la tourbière enregistre un assemblage pollinique correspondant à un paysage ouvert assez humide à Poaceae, Cyperaceae et fougères en bordure d'une aulnaie. La lande et les prairies sont régulièrement pâturées entraînant un premier pic de plantes rudérales.

Au sommet de la tourbe, de 87 à $60 \mathrm{~cm}$ (VAU-1b), l'enregistrement pollinique montre les arbustes pionniers, Corylus et Betula, colonisant les prairies et les landes alors qu'Alnus envahit une tourbière qui devient de plus en plus sèche.

\section{Palynozone VAU-2 $(60 \mathrm{~cm}-40 \mathrm{~cm})$}

La sédimentation limoneuse en arrière du barrage se met en place (VAU-2a) et la cariçaie se développe. Le 


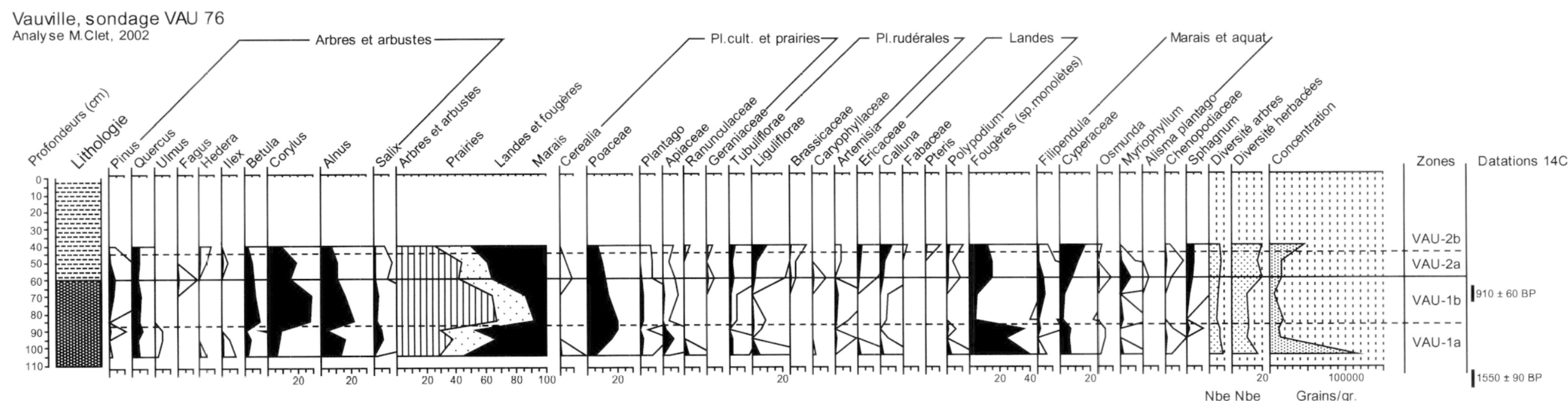

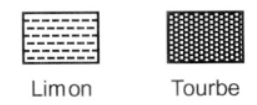

Figure 9 : Diagramme pollinique du sondage VAU 76 Figure 9 : Pollen percentage diagram - VAU 76. 
milieu devient plus humide et la présence d'eau libre est attestée par un pic de Myriophyllum. Puis, Alnus, Corylus et Betula commencent à décroître et les berges sont envahies par les fougères.

A $40 \mathrm{~cm}$ (VAU-2b), le paysage est ouvert même si quelques bosquets d'arbres et d'arbustes sont encore présents. Les Ericaceae indifférenciées et Calluna mais également l'ensemble des rudérales accroissent leur importance indiquant le développement des prairies, des cultures et surtout d'une lande régressive basse à bruyère.

\subsubsection{Les analyses micromorphologiques}

La colonne sédimentaire limoneuse a été prélevée dans sa totalité afin de réaliser des lames minces pour l'analyse micromorphologique (fig. 8). Celles-ci permettent de décrire les limons organiques de transition ayant fait l'objet d'analyses palynologiques (palynozone VAU 2) ainsi que le remplissage limoneux supérieur et le sol qui s'y développe. Il est possible de distinguer 3 séquences.

\section{Séquence de base $(80 \mathrm{~cm}-70 \mathrm{~cm})$}

L'organisation des sédiments est subhorizontale, marquée par l'alternance des lits de limons fins détritiques $(5 \mu \mathrm{m})$ dans un sédiment qui est globalement imprégné de matière organique et qui correspond à une argile limoneuse tourbeuse très riche en macrorestes végétaux (LM 5, photo. 1). L'organisation des dépôts et leur teneur indiquent le début du remplissage du réservoir du barrage par une sédimentation fine encore très organique dans un milieu aquatique comme l'indiquent également les analyses polliniques.

\section{Séquence intermédiaire $(70-20 \mathrm{~cm})$}

Vers le sommet, la sédimentation devient de plus en plus détritique. La disposition est toujours globalement subhorizontale mais la matrice est de plus en plus grossière. D'abord, clairement limoneuse $(7,5$ à $10 \mu \mathrm{m})$, elle comporte quelques grains de sable quartzeux jusqu'à $250 \mu \mathrm{m}$ (LM 4 et 3 , photo. 2). Vers le sommet, elle correspond à des limons et des sables quartzeux fins à moyens (15 à $400 \mu \mathrm{m}$ ) peu émoussés (LM 2, photo. 3). La fréquence des charbons de bois dans tous les horizons atteste vraisemblablement la pratique régulière du feu.

\section{Séquence sommitale $(20-0 \mathrm{~cm})$}

Elle montre une pédogenèse marquée par une décomposition peu avancée de la matière organique en milieu hydromorphe. Elle témoigne de l'atterrissement progressif de l'étang de retenue et sa conquête par des milieux palustres jusqu'au paysage actuel de saulaie.

\section{Discussion : l'évolution des paysages et l'empreinte des sociétés rurales}

Les analyses paléoenvironnementales permettent de nourrir une première discussion sur l'évolution des paysages de La Hague au cours des cinq derniers millénaires. Confrontées aux résultats des recherches archéologiques et historiques disponibles, elles permettent d'émettre les premières hypothèses sur le rôle des sociétés rurales

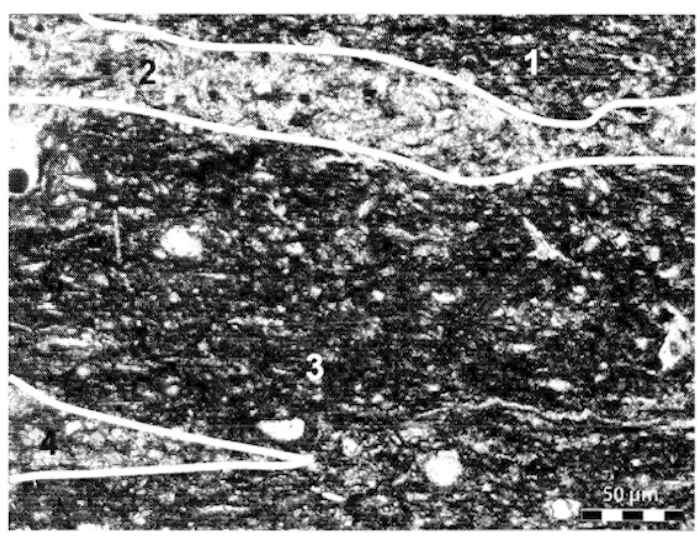

Photographie 1 : lame mince 5, sommet, LPNA.

Picture 1 : Thin section 5, up, PPL.

Photographie 1 : alternance de lits organiques riches en macrorestes végétaux et microcharbons ( 1 et 3 ) et de lits plus détritiques et limoneux (2 et 4).

Picture 1 : organic layers with macro-vegetal remains and micro-charcoal (1 and 3) alternating with loamy detritic layers (2 and 4).

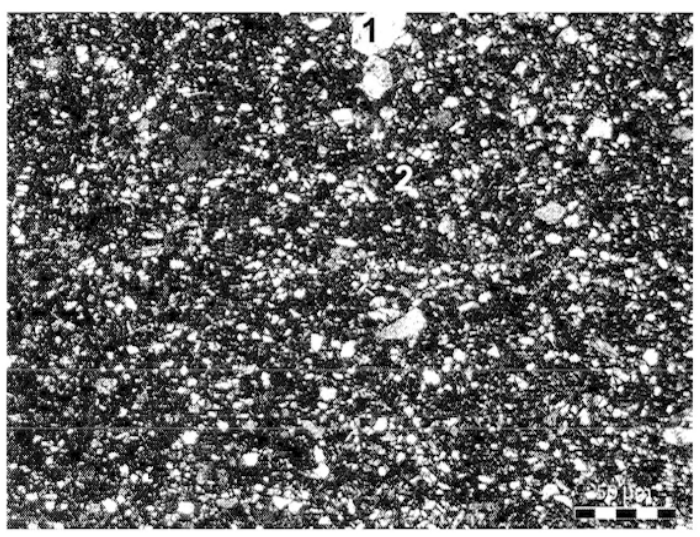

Photographie 2 : lame mince 4 , sommet, LPA.

Picture 2: Thin section 4, up, XPL.

Photographie 2 : limons à grains de sable épars (1), imprégnés de matière organique et de microcharbons (2).

Picture 2 : Loam with sand grains (1), organic matter and micro-charcoal (2).

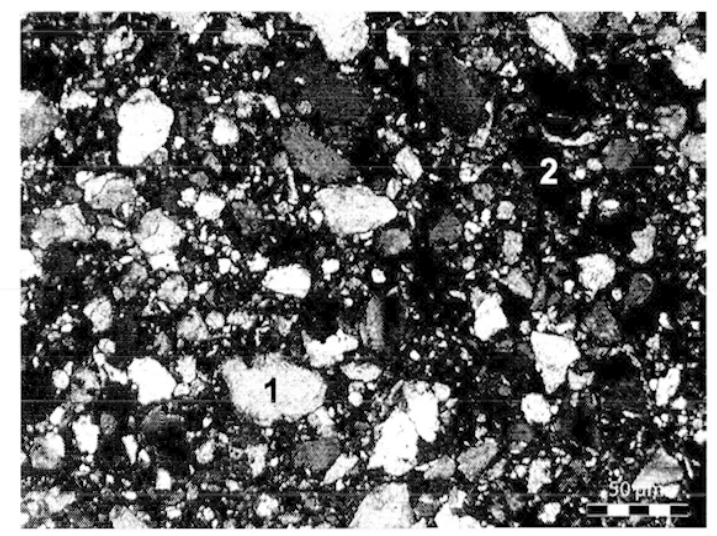

Photographie 3 : lame mince 2, base, LPA.

Picture 3 : Thin section 2, bottom, XPL

Photographie 3 : limons à sable quartzeux fin à moyen (1) et charbons épars (2).

Picture 3 : Loam with fine to middle size quartzitic sand (1) and charcoal (2).

L'échelle graphique représente $50 \mu \mathrm{m}$.

Graphic scale : $50 \mu \mathrm{m}$. 
dans les dynamiques paysagères depuis le Néolithique final. Les propositions de corrélation entre les analyses paléoenvironnementales et les événements chrono-culturels sont appuyées sur les âges calibrés obtenus pour établir la chronologie des archives sédimentaires (tab. 1) et sont rendues possibles par la grande stabilité des rythmes de la sédimentation organique dans les trois bas-marais étudiés.

\subsection{Les débuts de la sédimentation tourbeuse dans la plaine littorale de l'Anse Saint-Martin}

Dans l'Anse Saint-Martin, la sédimentation organique débute vers $4500-4100$ BP soit vers $3400-2600$ cal. av. J.-C. environ et se situe dans une période où l'initiation et le développement des tourbières littorales sont particulièrement actifs sur les deux rives de la Manche. Sur la rive sud, c'est entre 5000 cal. av. J.-C. et $1500 \mathrm{cal}$. av. J.-C. qu'apparaissent la plupart des marais et tourbières littorales connues dans le monde armoricain (Elhaï, 1963; Morzadec-Kerfourn, 1974; Marguerie, 1992). Dans le marais de Dol (baie du Mont-Saint-Michel), par exemple, la sédimentation tourbeuse s'amorce entre 4950 et 3900 cal. av. J.-C. mais elle connaît une nouvelle extension à partir de 3500-2700 cal. av. J.-C. (Morzadec-Kerfoun, 2002) synchrone de l'initiation de la tourbification observée à l'Anse Saint-Martin. Dans le sud de l'Angleterre, le développement de la sédimentation organique est remarquable entre 4200-3000 cal. av. J.C. en Cornouailles et vers 3700-1200 cal. av. J.-C. dans le Solent (Waller et Long, 2003). Pour l'Anse SaintMartin, comme pour l'ensemble des tourbières littorales fossilisées sur les estrans actuels ou fonctionnant encore aujourd'hui, la transgression flandrienne a joué un rôle moteur. La plupart de ces zones humides se sont développées alors que le niveau marin relatif était géographiquement proche du niveau marin contemporain favorisant le recul des cordons littoraux vers une situation proche de l'actuelle et la remontée des nappes phréatiques dans les plaines littorales.

Dans le détail, le développement des bas-marais littoraux dépend ensuite de facteurs plus complexes à saisir et à démêler (Charman, 2002) : l'évolution du niveau marin relatif en fonction des transgressions ou des régressions secondaires, les fluctuations climatiques conditionnant le niveau des nappes d'eau souterraines ou superficielles et l'action des Sociétés. Les informations sur les fluctuations relatives du niveau marin dans l'Anse Saint-Martin restent ténues. Après la mise en place du bas-marais, son déplacement vers l'intérieur parallèlement au recul du trait de côte constitue une évolution classique, fréquemment observée et bien documenté dans le Massif Armoricain (Marguerie, 1992). Elle est à l'origine de l'apparition des tourbes d'estran avec leurs bois fossiles qui ont servi de fondement à de nombreuses légendes de raz de marée et/ou de submersion de villes ou de villages. Dans l'Anse Saint-Martin, la légende de la «Vieille Eglise» rapporte l'histoire de la submersion de la forêt qui occupait la plaine littorale suite à l'ouverture, lors d'une tempête, d'une brèche dans le cordon littoral (Digard de Lousta, 1847). En fin de parcours la mer serait même venue détruire un édifice religieux mal protégé dont on suppose la localisation par la persistance d'un toponyme à l'ouest de l'Anse. D'après son rapporteur, cette légende relaterait un événement datant du VII ${ }^{c}$ siècle ap. J.-C. La coïncidence chronologique avec la fin de la sédimentation enregistrée sur l'estran est troublante. Cependant, la sédimentation tourbeuse a pu être tronquée de manière importante et le recul du cordon littoral a pu être plus tardif et s'établir par exemple entre le IX ${ }^{\mathrm{c}}$ et le XII ${ }^{\mathfrak{c}}$ siècle au moment de la transgression du Dunkerquien II bien attestée plus au sud dans la baie du Mont Saint-Michel (Morzadec-Kerfourn, 2002) et plus généralement dans le nord de la France (Louwye, Declercq, 1998). En outre, l'absence de preuves sédimentologiques d'une submersion marine rapide ne permet pas d'exclure l'hypothèse d'une asphyxie des formations arborées suite à un exhaussement du niveau de la nappe phréatique en arrière du cordon littoral.

La question du rôle des hommes dans le déclenchement de la sédimentation tourbeuse peut également être posée. En effet, le début de la sédimentation organique est caractérisé par de nombreux charbons de bois, et la présence de grains de pollen de Cerealia alors que les fouilles sur le site de la Jupinerie situé à 500 mètres environ du bas-marais montrent la présence d'une occupation dès le Néolithique moyen au moins et que les nombreuses traces d'occupation attestent la fréquentation régulière $\mathrm{du}$ bassin versant au Néolithique moyen et final (Marcigny et al., 2002, 2003) (fig. 2). Le rôle des défrichements dans l'accentuation du ruissellement et l'augmentation de l'hydromorphie dans les plaines alluviales est souvent mis en avant pour expliquer le développement des milieux palustres (Charman, 2002). Cependant, dans l'Anse Saint-Martin, les indices d'une activité anthropique au cours du Néolithique final sont trop ténus pour attribuer un rôle moteur aux hommes. En revanche, il n'est pas à exclure qu'ils aient pu jouer le rôle de facteur «aggravant».

\subsection{Les débuts du remplissage organique holocène dans les vallées}

Le début de la sédimentation organique conservée dans les fonds de vallées est plus récent. Il est daté de $2150+/$ 90, soit de la Tène au Haut-Empire (397 cal. av. J.-C.-50 cal. ap. J.-C.), dans la vallée de la Sainte-Hélène (sondage EPA, fig. 2) et de $1550+/-90$, soit du Bas-Empire à l'époque mérovingienne (261-617 cal. ap. J.-C.) dans la Grande-Vallée de Vauville.

Dans la vallée de la Sainte Hélène, le début du remplissage sédimentaire est caractérisé par un horizon organique immédiatement surmonté d'un sédiment limoneux. Il correspond à une époque où la construction d'un petit cône alluvial est attestée à l'aval dans la plaine littorale. Cette sédimentation limoneuse peut être reliée à une érosion diffuse des sols favorisant l'engorgement des fonds de talwegs dans un environnement en train de subir de profondes transformations sous l'action des hommes. Dans la Grande-Vallée de Vauville, la sédimentation tourbeuse se développe au sein d'un environnement déjà très anthropisé. Le dépôt d'une tourbe limoneuse peut 
sans doute être relié à une érosion diffuse des sols dans $l^{\prime}$ 'ensemble du bassin versant à partir du $\mathrm{III}^{\mathrm{c}}$ au VII ${ }^{\mathrm{c}}$ siècles ap. J.-C. Le rôle concomitant de la pulsation fraîche et plus humide du VI ${ }^{c}$ au VIII' siècle ap. J.-C. fréquemment attestée dans l'ensemble de l'Europe du NordOuest favorisant la remontée des nappes dans les fonds de vallée et le transfert des sédiments vers le talweg n'est pas à exclure non plus.

Dans le fond de la vallée de la Sainte-Hélène, ponctuellement (sondage EPA), une nouvelle phase de développement de la tourbe est attestée au cours du XIX ${ }^{c}$ siècle. Elle est due principalement à la déprise rurale : l'absence d'entretien du chenal artificiel de la Sainte-Hélène a entraîné son obstruction et la remontée locale du niveau de la nappe phréatique en arrière d'un chemin construit en remblai traversant le fond de la vallée. C'est ce même type d'évolution qui est enregistré derrière le barrage de la Grande-Vallée de Vauville où l'absence d'entretien de la retenue a entraîné son comblement et le développement de milieux tourbeux au cours des dernières décennies.

Dans ces différents cas, l'action des sociétés paraît importante. Elle est double et résulte soit de la mise en valeur agricole qui favorise l'érosion des sols et donc, dans un contexte de haut niveau des nappes phréatiques, le développement des milieux hydromorphes, soit de la mise en place ou de l'abandon d'aménagements hydrauliques contribuant à la gestion des écoulements de surface. Ces observations sont convergentes avec celles effectuées dans de nombreux fonds de petites vallées du Bassin de Paris, du Massif Armoricain et du Massif Central (Visset, 1979 ; Marguerie, 1992 ; Leroyer, 1997 ; Allée et al., 1997 ; Tourman, 2000).

\subsection{Les paysages pré- et proto-historiques}

La synthèse des données recueillies jusqu'à présent dans la péninsule de La Hague (fig. 10) permet de formuler de premières hypothèses sur l'évolution des paysages sous l'impact des sociétés depuis le Néolithique final.

Les analyses palynologiques réalisées à l'Anse SaintMartin mettent en évidence des paysages encore fermés au cours du Néolithique final dans la plaine littorale et sur ses bordures malgré une occupation humaine attestée dès le Mésolithique et au cours du Néolithique (Fosse et Vilgrain, 2000 ; Marcigny et al., 2002, 2003 ; Vilgrain et coll., 2003). Malgré la présence de grains de pollen de céréales et de plantes rudérales au cours du Néolithique final (base du sondage SM 60), les traces de défrichements et de mise en valeur agricole restent ténues et indiquent plutôt des milieux en mosaïque où la végétation arborée (chênes) côtoie des formations buissonnantes plus ou moins fermées dans les zones humides (aulnes), sur leur marge ou sur les versants encadrant la plaine littorale (noisetiers) et sans doute de rares clairières en prairies ou cultivées. Cependant le lieu d'enregistrement utilisé ne permet pas de conclure pour le paysage des échines qui arment la péninsule où les vestiges archéologiques sont très nombreux (fig. 2) même s'ils ont peu été étudiés. Il n'est pas impossible que dans cet environnement le recul du couvert forestier ait été localement plus précoce même si les données archéologiques suggèrent généralement pour l'ensemble de la péninsule une néolithisation assez tardive, vers le début du Néolithique moyen.

A partir de la fin du Néolithique final et tout au long de l'âge du Bronze, le sondage SM 60 de l'Anse SaintMartin suggère une ouverture modérée du paysage marqué par le développement des landes et des fougères. Celles-ci se développent aussi bien dans les milieux humides au détriment de l'aulnaie que sur les sols mieux ressuyés du pourtour de l'Anse aux dépens des noisetiers principalement. Cette situation est en accord avec les données archéologiques locales qui montrent que la péninsule a été le siège d'une grande activité durant cette période (levée de terre du Hague Dike, limites parcellaires probablement liées à des sites domestiques, très nombreux tumulus) y compris dans l'espace étudié (fig. 2). Elle correspond également à des occupations successives à La Jupinerie au cours du Bronze Ancien et du Bronze final. Au cours de cette dernière période les premières analyses sur les charbons de bois confirment d'ailleurs l'utilisation des chênes et des noisetiers pour le bois de feu (Marcigny et al., 2002, 2003).

L'évolution des paysages dans la plaine littorale de l'Anse Saint-Martin et sur ses bordures demeure modérée du milieu du $3^{\mathrm{c}}$ au milieu du $1^{\mathrm{cr}}$ millénaire av. J.-C. et la situation ne change véritablement que pendant la Tène où l'ouverture du paysage s'affirme. Certes, il semble que cela soit d'abord dû à une hausse du niveau de base favorisant le développement des zones humides et d'une nappe d'eau libre mais les traces d'activités agricoles sont ensuite plus franches. Les prairies connaissent alors un développement remarquable, les céréales voient leur importance croître et sont surtout enregistrées en continu alors que les plantes rudérales se développent. Parallèlement, le développement des apports détritiques dans les fonds de vallées et l'atterrissement partiel de la plaine littorale de l'Anse Saint-Martin témoignent d'une première phase notable d'érosion des sols.

\subsection{L'évolution des paysages au cours des deux der- niers millénaires}

Pour les deux derniers millénaires les informations sont plus nombrcuscs. Il est possible de s'appuyer sur les deux bassins versants étudiés.

Dans le bassin-versant de l'Anse Saint-Martin, l'ouverture maximale du paysage intervient au début de la période gallo-romaine. Dans les zones humides, l'aulne n'occupe plus qu'une place très secondaire concurrencée en partie par les espèces de milieux humides mais également par l'essor des pratiques agro-pastorales. La culture des céréales connaît un développement même si la pratique de l'élevage, responsable de l'accroissement des prairies, semble jouer le rôle moteur. Sur les versants escarpés et les sols mieux ressuyés, les espaces boisés caractérisés par les noisetiers et les chênes connaissent leur recul maximal. Ces évolutions du couvert végétal se reflètent également dans la poursuite des flux solides qui entraîne l'atterrissement définitif de la partie orientale de la plaine littorale de l'Anse Saint-Martin. Cette observation est convergente avec les données historiques disponibles qui témoignent de la prospérité des campa- 


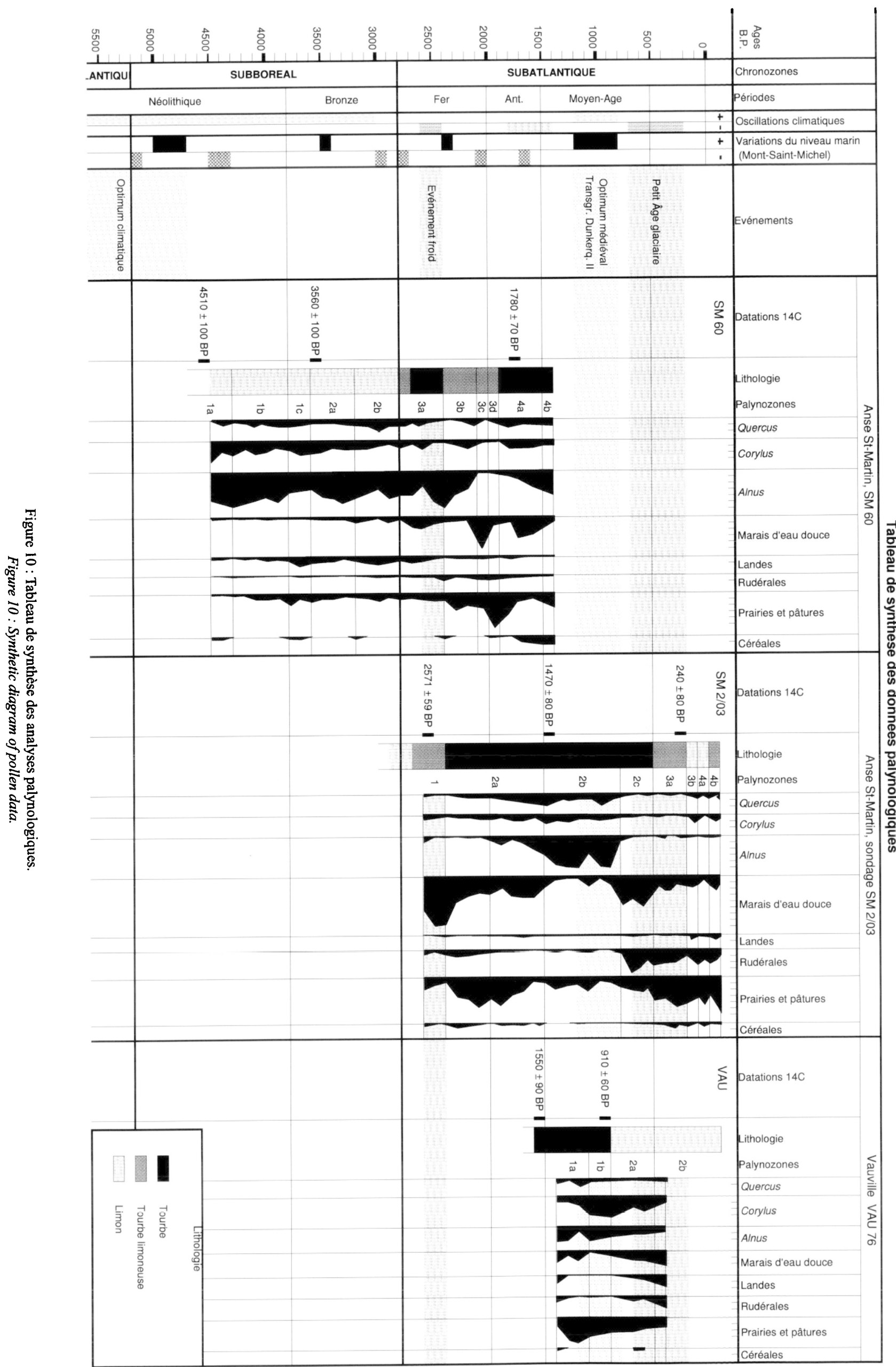


gnes bas-normandes du $\mathrm{I}^{\text {er }}$ au milieu du $\mathrm{III}^{\mathrm{c}}$ siècle ap. J.-C. (Deniaux, 2002) et les trouvailles archéologiques assez nombreuses pour La Hague à cette époque (PilletLemière et Levalet, 1989) y compris dans les bassins versants étudiés (fig. 2 et 7).

Dans l'Anse Saint-Martin, la fin de l'Antiquité et le début du Haut Moyen-Âge (fin du III ${ }^{\mathrm{e}}$ et IV $\mathrm{IV}^{\mathrm{e}}-\mathrm{V}^{\mathrm{e}}$ ap. J.-C.) sont marqués par un recul des marqueurs de l'anthropisation et en particulier le recul des prairies et une nouvelle phase de fermeture du paysage qui concerne aussi bien les milieux humides avec le développement de l'aulnaie que le reste du bassin versant avec le développement du noisetier et du chêne. Cette évolution cesse aux alentours des $\mathrm{XI}^{\mathrm{c}}-\mathrm{XI}{ }^{\mathrm{c}}$ siècles. Dans la Grande-Vallée de Vauville, le milieu nettement anthropisé au début du Haut MoyenAge avec une extension notable des prairies et des landes connaît une évolution semblable qui semble plus tardive, débutant vers le $\mathrm{VII}^{\mathrm{e}}$ siècle ap. J.-C., mais qui prend fin également vers les $\mathrm{XI}^{\mathrm{c}}-\mathrm{XII}^{\mathrm{c}}$ siècles. Cette évolution qui affecte les deux bassins versants peut probablement être interprétée comme le résultat d'une déprise rurale. Celleci est peut-être localement le reflet des difficultés économiques qui s'amorcent avec les invasions de la deuxième moitié du III ${ }^{\mathrm{c}}$ siècle et du $\mathrm{IV}^{\mathrm{k}}$ siècle et se poursuivent souvent jusqu'à la fin du VII' siècle ap. J.-C. (Bauduin et $a l .$, 2002). La reprise postérieure attestée d'une manière générale en Normandie ne semble pas se traduire par un changement dans les paysages et peut-être faut-il y voir l'empreinte des troubles des $\mathrm{IX}^{\mathrm{c}}$ et $\mathrm{X}^{\mathfrak{c}}$ associés aux poussées bretonnes et aux invasions normandes en particulier dans cette partie de la Basse-Normandie ; même si aujourd'hui l'idée d'une rupture brutale dans l'occupation des sols et les structures de peuplement est abandonnée (Bauduin et al., 2002).

A partir des $\mathrm{XI}^{\mathrm{c}}-\mathrm{XII}^{\mathrm{c}}$ siècles, la mise en valeur du milieu s'affirme à nouveau et semble perdurer jusqu'à la fin du $\mathrm{XX}^{\mathrm{c}}$ siècle. Les paysages de prairies pâturées ou fauchées et les parcelles cultivées se développent dans des espaces où la gestion de l'eau est de plus en plus maîtrisée comme le montre l'exemple du moulin de Vauville attesté dès le XII ${ }^{\mathrm{c}}$ siècle. Dans la Grande-Vallée de Vauville et à un degré moindre sur les pourtours de l'Anse Saint-Martin, les landes basses régressives à Ericaceae prennent de l'ampleur alors qu'apparaissent les ajoncs (Fabaceae). Dans la Grande-Vallée de Vauville, le nombre important de charbons trouvé dans la séquence détritique du remplissage du barrage permet d'évoquer le rôle du feu dans l'apparition et l'entretien de la lande basse alors que l'augmentation progressive de la granulométrie des formations sédimentaires piégées derrière le barrage indique une accentuation du ruissellement mais aussi corrélativement une dégradation des profils pédologiques à partir du milieu du XII ${ }^{\mathrm{c}}$ siècle au moins.

\section{Conclusion}

Dans la péninsule de La Hague, l'évolution des paysages est sous l'emprise des sociétés au moins depuis la charnière Néolithique final - âge du Bronze. Cela est en concordance avec les observations réalisées dans les tourbières littorales du nord et de l'ouest de la Bretagne (Morzadec-Kerfourn, 1974 ; Marguerie, 1992) ou les tourbières de plateaux du sud de l'Angleterre (Caseldine, 1999 ; Gearey et al., 2000b ; Simmons, 2003). L'ouverture des paysages reste néanmoins modérée tout au long de l'âge du Bronze même si les premiers paysages de landes à fougères sont repérés. Cependant l'installation et la généralisation des formations prairiales et de landes océaniques emblématiques de la péninsule semblent bien plus tardives. Ces premiers résultats sont en retrait par rapport aux évolutions constatées dans certains espaces littoraux du monde armoricain où le recul forestier peut être notable et $\mathrm{s}$ 'accompagner de la mise en culture dès l'âge du Bronze (Marguerie, 1992) mais surtout par rapport à ce qui a été montré en Grande-Bretagne et en particulier dans le sud-ouest de l'Angleterre où l'on observe le déclin généralisé du couvert forestier en relation avec le développement de l'élevage et parfois même la mise en place de système de parcellaire (Caseldine, 1999 ; Gearey et al., 2000 ; Simmons, 2003).

L'ouverture des paysages qui témoigne d'une déforestation intense et de la mise en place de prairies et des cultures intervient principalement au cours de la Tène et de la période gallo-romaine. Les premières données sur le développement des landes basses régressives montre qu'il est probablement attribuable localement à la fin de l'époque médiévale. Il serait contemporain des transformations constatées dans le Bodmin Moor (Gearey et al., $2000 \mathrm{~b}$ ) et dans le nord-mayennais (Barbier, 1999) mais plus tardif que dans de nombreux espaces armoricains (Marguerie, 1992). Cette évolution peut être mise en relation localement avec le développement de l'érosion et la dégradation progressive des sols mis à nu du fait des défrichements qui s'amorcent au cours des époques préet proto-historiques, comme dans l'ensemble du monde armoricain (Gebhardt, 1990), mais qui s'intensifient surtout au cours de l'époque médiévale.

Malgré leur aspect partiel, les premières recherches menées dans la péninsule de La Hague montrent une cohérence d'ensemble avec les observations réalisées dans les Finistères océaniques de l'Ouest européen même si des particularités peuvent être notées. De nouvelles recherches paléoenvironnementales sont nécessaires pour affiner le modèle chronologique d'évolution des paysages de la péninsule et préciser l'extension des changements observés dans l'Anse Saint-Martin et à Vauville. Elles seront menées en collaboration avec les fouilles archéologiques afin de mieux cerner le lien entre la gestion des milieux par les sociétés du Néolithique à l'époque moderne et l'évolution des paysages.

\section{Remerciements}

Cette recherche a pu bénéficier du soutien financier et logistique de la Communauté de Communes de La Hague et d'unc ACI « Jeunes chercheurs » du Fonds National pour la Science intituléc « Gestion de l'cau et dynamiques des paysages du Néolithique à nos jours : étude des basses vallées côtic̀res dans le nord-ouest de la France ». Nous tenons plus particulièrement à remercier Florent Bellec pour son aide précieuse et constante. Nous remercions également le Groupe de Recherches Archćologiques du Cotentin et le Scrvice Régional de l'Archćologique de Basse-Normandic. 


\section{Références bibliographiques}

ALLEE, P., DIOT, M.-F., DURIEUX, J.-F., REYNET, J.-M., VALADAS, B., 1997 - Trois mille ans d'cnregistrement sćdimentaire dans les fonds de vallons des bas-plateaux limousins. La dynamique des paysages protohistoriques, antiques médiévaux et modernes, Actcs des XVII ${ }^{c}$ Rencontres Internationales d'Archéologic ct d'Histoire d'Antibes, APDCA, Sophia Antipolis, 365-387.

BARBIER, D., 1999 - Histoire de la Végétation du nord-mayennais de la fin du Weichsélien à l'aube du XXIe siècle. Mise en évidence d'un Tardiglaciaire armoricain. Interactions Homme-Milieu. Groupc d'Etude des Milieux Naturels, Nantes, 2 vol., 284 p.

BAUDUIN, P., JARRY, T., LORREN, C., 2002 - La Futurc Normandic du III $^{c}$ au X $\mathrm{X}^{\mathrm{c}}$ siècle. In Deniaux, E., Lorren, C., Bauduin, P., Jarry, T., La Normandie avant les Normands, Ouest-France, Rennes, 198-415.

BILlARD, C., CLET-PELLERIN, M., LAUTRIDOU, J.P., 1995 Un site protohistorique littoral dans le Hâvre de la Vanlée, à Lingreville et Bricqueville-sur-mer (Manche). Rev. Archéo. Ouest, 12, 71-110.

BROWN, A. G., 1999 - Characterising Prehistoric lowland environments using local pollen assemblages, Quaternary Proceedings 7, 585-594.

CAMPBELL, J., 2000 - Holocene Palaeoenvironments of Guernesey and Alderney, Channel Islands, Unpublished $\mathrm{PhD}$ thesis, Coventry University, $367 \mathrm{p}$

CAMPBELL, J., JAMES, H.C.L., RENOUF, J.T., 2001 - Changing Holocene Environments in Alderney, Channel Islands. Geoscience in south-west England, 10, 154-159.

CASELDINE, C. J., 1999 - Archacological and Environmental Change on Prehistoric Dartmoor : current understanding and future directions. Quaternary Proceedings 7, 575-584.

CHANCEREL,A., GHESQUIÈRE, E., MARCIGNY, C., VERRON, G., avec la collaboration de DUPRET, L., LE GALL, J., 1996 - Lc site Néolithique des «Treize Vents» à Herqueville (Manche). Bulletin de la Société Préhistorique Française, 93, 2, 241-249.

CHARMAN, D., 2002 - Peatlands and Environmental Change. Wilcy and Sons Ltd, Chichester, $301 \mathrm{p}$.

CLET-PELLERIN, M., 1985 - Synthèse palynologique de quelques sites archéologiques de Normandie depuis le Néolithique. In Palynologie Archéologique, Actes des Journćes du 25, 26, 27 janvier 1984, Notes et Monographies techniques, 17, CNRS, Paris, 425-442.

CLET-PELLERIN, M., DUPEUBLE, P. A., HELLUIN, M., LEFEBVRE, M., PELLERIN, J. et coll., 1987 - Les formations marines et continentales du Pléistocène Récent dans la région d'AsnellesSaint-Côme de Fresné (Calvados). Bulletin du Centre de Géomorphologie, 32, Caen, 71-98.

CLIQUET, D., 1994 - Le gisement paléolithique moyen de Saint-Germain-des-Vaux/Port Racine (Manche) dans son cadre régional. Essai palethnographique. Editions ERAUL, Liègc, 63, 2 vol., 644 p.

COUTARD, S., 2003 - Formations quaternaires en bordure d'une mer épicontinentale, la Manche. Tectonique, eustatisme, climat et occupations humaines. Exemple du Val de Saire (Normandie, France). Université de Caen, Thèse Doctorat, $446 \mathrm{p}$.

COUTARD, S., CLET-PELLERIN, M., à paraître - Évolution de la sédimentation et de la végétation pendant l'Holocène dans les marais arrière-littoraux du Val de Saire (Cotentin, Normandie). Actes de la Table Ronde en l'honneur de René Neboit-Guilhot. Collection «Nature et Sociétć», Presses Universitaires Blaise Pascal, Clermont Ferrand, 6 p.

DENIAUX, E., 2002 - L'Antiquité. In Deniaux, E., Lorren, C., Bauduin, P., Jarry, T., La Normandie avant les Normands, Ouest-Francc, Rennes, 0-198.

DIGARD de LOUSTA, 1847 - Légende de l'inondation de la Forêt de Saint-Martin. Mémoire de la Société Académique de Cherbourg, 112-116.
EDWARDS, K., 1999 - Palynology and People : observations on the British records. Quaternary Proceedings, 7, 531-544.

ELHAÏ, H., 1963 - La Normandie occidentale entre la Seine et le golfe normano-breton. Etude morphologique. Thèse dc doctorat, Bordeaux, Bière, 581 p.

FOSSE, G., VILGRAIN, G., 2000 - Le sitc de Jardehcu-La Gravette à Digulville (Haguc). Inventaire archéologique sommaire et structures de combustion préhistoriques. L'archéologie dans la Manche: fouilles et recherches récentes (1990-1999). Etudes ct Documents, Collection de la Socićté d'Archćologic et d'Histoirc de la Manche, Saint Lô, 125-131.

GEAREY, B. R., CHARMAN, D. J., KENT, M., 2000a - Palacoccological evidence for the Prehistoric settlement of Bodmin Moor, Cornwall, Southwest England. Part I : The status of woodland and carly human impacts, Journal of Archaeological Science, 27, 423-438.

GEAREY, B. R., CHARMAN, D. J., KENT, M., 2000b - Palacoccological evidence for the Prehistoric settlement of Bodmin Moor, Cornwall, Southwest England. Part II : Land Use Changes from the Ncolithic to the Present, Journal of Archaeological Science, 27, 493-508.

GEBHARDT, A., 1990 - Evolution du paléopaysage agricole dans le Nord-ouest de la France, apport de la micrumorphologie. Thèsc dc doctorat, Rennes I, $191 \mathrm{p}$.

GERARD, F., 1972 - Les noms de lieux du canton de Beaumont-Hague. Publication multigraphiée, fasc. 14, Ecole des Chartes, Paris, 432 p.

GHESQUIÈRE, E., LEFÈVRE, P., MARCIGNY, C. et SOUFFI, B., 2000 - Le Mésolithique moyen du Nord-Cotentin, Basse-Normandie, France, International Series, British Archacological Reports (BAR), Oxford, $\mathrm{n}^{\circ} 856,292 \mathrm{p}$.

HAUGUEL, J.-C., LECOINTE, A., 1996 - Les landes de Vawille, expertise botanique. Rapport au conservatoire du Littoral, $45 \mathrm{p}$.

LEROYER, C., 1997 - Homme, climat, végétation au Tardi-et Postglaciaire dans le Bassin Parisien, apports des études palynologiques des fonds de vallées. Thèse de doctorat, Paris 1,2 vols, $550 \mathrm{p}$.

LESPEZ, L., CLET-PELLERIN, M., LEVALET, F., BELLEC, F., DAVIDSON, R., 2003 - L'apport des archives naturelles dans la compréhension de l'évolution des paysages protohistoriques et historiques normands. L'exemple de la péninsule de La Hague, Manche. Annales de Normandie, Congrès des Sociétés Historiques et Archéologiques de Normandie, 8, 77-93.

LETTERLE, F., VERRON, G., 1986 - Un site d'habitat campaniforme à Digulville (Manche), Revue Archéologique de l'Ouest, suppl. 1, 237-252.

LOUWYE, S., DECLERCQ, E., 1998 - Relative water level change in the intracoastal zone of Belgium and Northern France over the last 2500 years. Boreas, 27, 162-177.

MARCIGNY, C., JUHEL, L., LESPEZ, L. et GHESQUIÈRE, E., et coll., 2002 - Omonville-La-Petite, L'abri de la "La Jupinerie», Manche), Fouille d'un abri sous-roche occupé du Néolithique ancien à la fin de l'âge du Bronze. Rapport de Fouille 2002, DRAC-Basse Normandie, INRAP, Conseil Général de la Manche, $28 \mathrm{p}$.

MARCIGNY, C., JUHEL, L., et GHESQUIÈRE, E., et coll., 2003 Omonville-La-Petite, «La Jupinerie», Manche), Fouille d'un abri sous-roche occupé du Néolithique ancien à la fin de l'âge du Bronze. Rapport de Fouille 2003, DRAC-Basse Normandic, INRAP, Conseil Général de la Manchc, $44 \mathrm{p}$.

MARGUERIE, D., 1992 - Evolution de la végétation sous l'impact humain en Armorique du Néolithique aux périodes historiques. Travaux du laboratoire d'Anthropologie de Rennes, 40, Université de Rennes $1,313 \mathrm{p}$.

MORZADEC-KERFOURN, M.-T., 1974 - Variations de la ligne de rivage armoricaine au Quaternaire. Analyses polliniques de dépôts organiques littoraux. Mem. Soc. Gcol. Min. Bretagne, 17, Rennes, $208 \mathrm{p}$. 
MORZADEC-KERFOURN, M.-T., 2002 - La sédimentation holocène dans la partie occidentale de la baic du Mont-Saint-Michel : l'évolution du marais de Dol-de-Bretagne. In Bonnot-Courtois, C., Calinc, B., L'homer, A., Le Vot, M. (cds), La baie du Mont-SaintMichel et l'estuaire de la Rance, environnements sédimentaires, aménagements et évolutions récentes, CNRS, EPHE, Total-Elf-Fina, Mémoire 26, Pau, 153-164.

STUIVER, M., REIMER, P. J., BARD, E., BECK, J. W., PLICHT, J., SPURK, M., 1998 - Radiocarbone, 40, 1041-1083.

PILLET-LEMIERE, J., LEVALET, D, 1989 - La Manche, Carte archéologique de la Gaule, Académic des Bclles Lettres, Paris, 136 p.

PROVOST, M., LECOINTE, A., 1985 - Inventaire des landes et des pelouses calcicoles du Calvados et de la Manche. DRAE Bassc-Normandie, CREPAN, $20 \mathrm{p}$.
SIMMONS, I.G., 2003 - The Moorlands of England and Wales, an environmental history $8000 B C$ to $A D 2000$, Edinburgh University Press, $414 \mathrm{p}$.

TOURMAN, A., 2000 - La mise en place des tourbières dans le bassinamont de la Loire, approche paléogéographique et exploitation des datations par le radiocarbone, Mémoire de DEA, Universitć de Saint-Etienne, $59 \mathrm{p}$.

VII.GRAIN, G. et coll, 2003 - Digulville, La Gravette-Falguic̀res. Bilan scientifique 2001. DRAC -SRA Bassc Normandic, 64-65.

VISSET, L., 1979 - Recherches palynologiques sur la végétation Plćistocène et Holocène de quelques sites du district phytogéographique de Bassc-Loire. Bulletin de la Société des Sciences naturelles de l'Ouest de la France, supplément H.S., Nantes, 282 p.

WALLER, M. P., LONG, A. J., 2003 - Holocene coastal cvolution and sea-level change on the southern coast of England : a revicw. Journal of Quaternary Science, 18, 3-4, 351-359. 